ÉGYPTE monde arabe

\section{Égypte/Monde arabe}

4 | 1990

Démocratie et démocratisation dans le monde arabe

\title{
Les législatives égyptiennes ou la politique entre clientélisme et citoyenneté
}

Dossier de presse

Iman Farag

\section{OpenEdition}

\section{Journals}

Édition électronique

URL : https://journals.openedition.org/ema/353

DOI : 10.4000/ema.353

ISSN : 2090-7273

Éditeur

CEDEJ - Centre d'études et de documentation économiques juridiques et sociales

Édition imprimée

Date de publication : 31 décembre 1990

ISSN : 1110-5097

\section{Référence électronique}

Iman Farag, «Les législatives égyptiennes ou la politique entre clientélisme et citoyenneté », Égypte/ Monde arabe [En ligne], 4 | 1990, mis en ligne le 08 juillet 2008, consulté le 07 juillet 2022. URL : http:// journals.openedition.org/ema/353 ; DOI : https://doi.org/10.4000/ema.353

Ce document a été généré automatiquement le 7 juillet 2022.

Tous droits réservés 


\section{Les législatives égyptiennes ou la politique entre clientélisme et citoyenneté}

Dossier de presse

Iman Farag

De la politique comme spectacle, qui n'a pas

l'expérience?

Alessandro Pizzorno ${ }^{1}$

129 novembre et 6 décembre 1990 : élections législatives en Égypte. 16 millions d'inscrits sur les listes électorales, répartis sur 222 circonscriptions et 236167 bureaux de vote ; ils doivent élire 444 députés parmi les 2681 candidats, chaque circonscription étant représentée par deux élus dont au moins un ouvrier ou un paysan. Les candidats se répartissent entre le Parti national démocrate (PND) au pouvoir, 444 candidats, le Rassemblement progressiste (RP), 52 candidats, le Parti al-Umma, 22 candidats, et les nouvelles organisations: Misr al-Fatat (Jeune Égypte), le Parti al-Ittihadi (unioniste) et le Parti des Verts, outre les candidatures indépendantes autorisées par la nouvelle loi électorale. Le néo-Wafd, le Parti du Travail, les Frères Musulmans et le Parti al-Ahrar (Libéraux) ont décidé de boycotter les élections².

2 Le résultat final donnera au PND 79,6\% des voix, soit 348 sièges, 19\% aux indépendants, soit 83 sièges dont 23 aux candidats membres des «partis du boycott» (14 au Wafd, 8 au Parti du Travail, 1 aux Libéraux) ; enfin 1,4\% au RP, soit 6 sièges ${ }^{3}$.

3 Un politologue sérieux traiterait de ces résultats, des grands mouvements d'opinion dont ils sont révélateurs, des positions des forces en présence et des perspectives d'avenir. Il s'intéresserait aux programmes politiques, à la confrontation des « idéauxlogies ", aux rouages de la machine électorale ou encore aux logiques culturelles et sociales sous-jacentes à ces éléments. Peu lui importerait que ces élections, moments d'un évergétisme princier, soient vécus dans certains quartiers comme une réjouissance populaire (outre qu'il s'agit quelquefois d'un jour chômé), que la campagne électorale fasse la joie des badauds, le bonheur des fabricants de calicots et 
des calligraphes, la fortune des agences de location de chapiteaux et de haut-parleurs, ou encore celle des "coursiers en élections", justiciers et bagarreurs à la tête de bandes de quartiers, chargés d'intimider les concurrents, d'organiser les meetings, les banquets et les manifestations de soutien et, le grand jour, de faire la sentinelle devant les bureaux de votes, si ce n'est à l'intérieur...

Le politologue jugerait-il intéressant de s'arrêter devant une multitude de slogans, nouveaux et récurrents à la fois, qui chantent les vertus d'Untel et que la gouaille populaire s'empressera de retourner - par un pastiche plus ou moins subtil - contre le malheureux candidat?

5 Il ne m'a pas paru nécessaire, quant à moi, de privilégier les dimensions «supposées nobles» de l'événement, pour une simple raison: c'est que la politique "à l'égyptienne ", c'est tout cela à la fois. Cela l'est d'autant plus que depuis 1952, les élections législatives n'ont donné lieu à aucune alternance du pouvoir, qu'elles se caractérisent par l'unanimisme que l'on sait, par l'emprise des appareils administratifs - quand ils ne sont pas policiers - et que le pourcentage réel des votants défie toute logique mathématique de «représentation de la nation ».

6 Mais ce ne sont pas là des raisons pour en sous-estimer la valeur heuristique. Bien au contraire, les élections nous en apprennent long sur ce. que d'aucuns appellent « les recompositions du champ politique" et sur les mille façons d'asseoir ou de contrecarrer une légitimité politique. Au-delà du remue-ménage qui agite la "scène politique ", il se déroule dans la salle un autre spectacle...

Conjonctures : un parlement à enterrer

7 C'est dans un contexte bien spécifique et selon des modalités nouvelles que se sont déroulées les élections du vingt-et-unième parlement de l'histoire égyptienne. Le 19 mai de cette même année, la Haute Cour constitutionnelle frappait d'inconstitutionnalité l'article 5 bis de la loi électorale sur la base de laquelle avait été élue l'Assemblée et qui défavorisait les candidatures indépendantes par rapport aux listes des partis. Le 26 septembre, trois réformes électorales modifiaient le découpage des circonscriptions, les faisant passer de 176 à 222, et rétablissaient le scrutin uninominal et lés candidatures indépendantes ${ }^{4}$. Le 11 novembre 1990, 8,8 millions d'électeurs - soit $94,34 \%$ des inscrits, selon les sources officielles - approuvaient par référendum la dissolution de l'Assemblée du Peuple, contre 5,66\%, avec un taux de participation annoncé de $58,66 \%$. C'était, sur 17 référendums, la troisième consultation relative à une dissolution du parlement. Quelques instants avant l'annonce des résultats, Rifat al-Mahgub, président de l'Assemblée, était assassiné.

8 A la veille du référendum, la presse officieuse donnait le ton. Salah Muntasir écrivait dans Al-Ahram : «Le référendum pourrait-il aboutir à un « non » ? J'affirme sans hésiter que tout est possible. (...). Je ne pense pas outrepasser la vérité en disant que - sauf antipathies personnelles pour certains députés - le sentiment général va dans le sens de la dissolution de l'Assemblée. $»^{6}$

9 Et si le peuple refusait la dissolution? C'est la question que pose un hebdomadaire au président du Conseil supérieur de la magistrature :

La mesure prise par le président de la République tentait de s'adapter à l'opinion.

(...) Le refus est un cas de figure théorique qu'on ne peut pour autant exclure, et qui vaut pour tout référendum proposé par le président de la République en vertu du droit que lui donne la Constitution. (...) Cela dit, la direction politique qui a proposé le référendum a sondé l'opinion et n'a pas envisagé ce cas de figure. ${ }^{7}$ 

caduc dans la mesure où la Haute Cour s'était déjà prononcée sur l'inconstitutionnalité de l'Assemblée. Ainsi l'organe du Wafd écrivait-il, sous le titre "Pourquoi les référendums égyptiens ont-ils mauvaise réputation » :

Leurs résultats sont prévus à l'avance et conformes à la volonté gouvernementale. (...) Ils ont été pratiqués avec une fréquence et une désinvolture telles qu'ils sont devenues monnaie courante. (...) Selon la Constitution, référendums et élections doivent être soumis au contrôle effectif de la magistrature. Or le pouvoir législatif délègue cette tâche importante à des fonctionnaires sans scrupules. (...) Le ministre de l'Intérieur a déclaré dans une interview qu'il voterait pour la dissolution, voulant ainsi peser sur les décisions de l'opinion alors que, censé veiller sur la régularité des élections, il devrait adopter une position de neutralité (...) Ce référendum est une mesure purement formelle, dont le seul but est de minimiser la portée du jugement émis par la Haute Cour constitutionnelle. ${ }^{8}$

Dans le même ordre d'idées, des réserves sont émises à l'égard de la nouvelle loi électorale, "remake de la loi frappée d'inconstitutionnalité » selon Al-Wafd: "A l'exception $\mathrm{du}$ rétablissement $\mathrm{du}$ scrutin uninominal, toutes les contraintes demeurent. $\rrbracket^{9}$ Ma'mun al-Hudaybi, ex-président du groupe parlementaire des Frères musulmans, ajoute que « la consultation électorale est toujours soumise à l'autorité du ministre de l'Intérieur, de A à Z. $»^{10}$

Son homologue wafdiste souligne que « la nouvelle toi néglige les garanties minimales revendiquées par l'opposition, qui consistaient à enlever des listes électorales les noms des personnes décédées ou émigrées, d'y inscrire obligatoirement tous les jeunes audessus de 18 ans et de faire connaître les listes et les adresses des bureaux de vote plusieurs semaines avant les élections. $»^{11}$ II souligne par ailleurs l'inégalité des chances d'accès aux médias. Dans Al-Wafd toujours, on retrouve ce verdict catégorique : « Tant qu'existera une Constitution de conception totalitaire et que durera la loi d'urgence appliquée depuis dix ans, il ne sert à rien de réclamer des élections propres. ${ }^{12}$ Ainsi la grande consultation était-elle placée dès le départ sous le signe du malentendu, cela en dépit des remaniements de la loi électorale.

Nouveaux éléments

Quant à l'application d'un scrutin strictement uninominal et l'autorisation des candidatures indépendantes, elles ont été appréciées de manières différentes. Pour Mayo, organe du PND, le retour au scrutin uninominal « donne une saveur nouvelle aux élections et enrichit la compétition électorale. (...) Il permet de faire accéder à l'élite. $»^{13}$ D'autres opinions sont plus nuancées :

14 «Seul le scrutin de liste permettait d'ouvrir la voie à des personnalités de valeur peu rompues au jeu électoral et à ses techniques. Toutefois, son application en Égypte entraînait souvent des confusions dans la mesure où ouvriers et paysans devaient représenter au moins 50\% des candidatures, selon la Constitution. Autre inconvénient, ce mode de scrutin donnait lieu à de longues listes, jusqu'à 13 candidats parfois, alors qu'il aurait été possible d'augmenter lé nombre de circonscriptions et de limiter les listes à 5 candidats, réduisant ainsi les conflits entre ceux qui sont en tête de liste et ceux qui arrivent en queue du peloton. $»^{14}$

15 Ces deux facteurs modifiaient largement le jeu et les enjeux des forces politiques, sinon les modalités de la bataille électorale, les affinités et les programmes politiques - ces derniers étant déjà peu significatifs - perdant de leur importance au profit de l'insertion locale des candidats. Dans une telle configuration, le politique tend plus que

Égypte/Monde arabe, 4 | 1990 
jamais à se construire en tant que médiation entre intérêts particuliers et intérêts publics, et les contours de la chose politique s'en trouvent modifiés. Par ailleurs, la conjoncture «scrutin uninominal et candidatures indépendantes » laissait croire à un renouvellement éventuel, même s'il n'est que partiel, de la classe politique, notamment par le biais de l'intégration institutionnelle des exclus privés de représentation politique, Frères musulmans et nassériens; les premiers y étant parvenus par le truchement d'alliances électorales successives, les seconds rejetés dans une «clandestinité qui n'en est pas une ». En ce sens et comme le note avec raison Alain Roussillon, l'enjeu de cette bataille électorale, tout comme celui de la loi électorale, n'était pas tant l'élection d'une majorité que les modalités d'élection d'une opposition. 15

16 Les nouvelles dispositions de la loi électorale allaient marquer de manière significative tant le résultat des élections que le déroulement de la campagne électorale, campagne à double facette: celle, pittoresque et bariolée, qui se déroulait dans la rue et dont la presse a largement rendu compte - en évitant toutefois de s'attarder sur les affinités politiques « dérangeantes » de certains candidats de gauche et nassériens - et celle qui se déroulait exclusivement entre pouvoir et opposition par organes de presse interposés, et dont les principaux protagonistes, du côté de l'opposition, étaient les partis qui avaient décidé le boycott. Il n'est pas exagéré de dire que ceux qui furent les plus présents dans la seconde, celle des journaux, ont brillé par leur absence dans la première, celte des rues, qui elle obéissait à d'autres lois et dont les enjeux concrets se définissaient « à chaud », sur le terrain, en marge du jeu politique national.

Le boycott : des raisons aux prétextes

17 La décision de boycott venait-elle semer des embûches sur le chemin d'un pouvoir décidé à amorcer une ouverture politique? Si oui, quelles en étaient les limites? Et de toute façon, comment évaluer les arguments avancés par les partisans du boycott alors qu'ils avaient déjà participé au jeu électoral dans des contextes beaucoup plus défavorables?

18 L'absence des garanties revendiquées par l'ensemble de l'opposition est l'argument principal avancé par les partisans du boycott. Il s'agit notamment, pour l'opposition, de l'absence d'un contrôle réel des juges sur les processus de vote et de dépouillement, contrôle institué par la Constitution et bafoué - selon l'opposition - par le pouvoir exécutif. Il n'est pas sans intérêt de souligner ici le crédit que détient la magistrature égyptienne auprès des forces de l'opposition, comme héritière d'une tradition libérale "réelle» et comme seule instance d'arbitrage digne de confiance, crédit auquel ne peuvent prétendre les pouvoirs législatif et exécutif dans une société où le pouvoir voire la chose publique en général - est perçu comme moyen de réalisation des intérêts particuliers, « corrompu par essence » si l'on peut dire.

Les partisans du boycott expliquent, dans un texte intitulé « Déclaration des partis de l'opposition » - même s'ils n'en représentent qu'une partie - leur décision :

Le pouvoir a amendé la loi électorale dans le plus grand secret et sans que l'opinion puisse en débattre. Il s'est avéré par ailleurs qu'hormis l'instauration du scrutin uninominal, aucune des garanties réclamées par l'opposition, les forces nationales, les syndicats et les clubs d'enseignants n'a été prise en compte. La loi a négligé également le contrôle des juges sur les élections, stipulé par la Constitution. Qui plus est, le redécoupage électoral a été effectué contre toute logique, selon les vœux du parti au pouvoir et de ses candidats. Tout cela confirme le fait que le pouvoir ne tenait nullement à des élections libres et neutres, d'autant que celles-ci se 
déroulent sous la loi d'urgence. Dans la poursuite de ses propres objectifs, le pouvoir est allé jusqu'à perdre de vue qu'il présentait là les preuves d'inconstitutionnalité du futur parlement. (...) L'opposition, croyant de manière inébranlable en la nécessité d'un système démocratique dans le vrai sens du terme afin de faire face aux problèmes et aux crises auxquelles est confrontée la société, et refusant de tromper la nation en prenant part à une démocratie de façade en ces temps d'épreuve, a pris la décision de boycotter les élections du 29 novembre. Les membres qui iront à rencontre de cette décision seront exclus, et seront exclus également ceux qui accepteront d'être nommés par le gouvernement. Signataire : Parti al-Wafd, Parti du Travail, Frères Musulmans, Parti des Libéraux. ${ }^{16}$

20 A ces arguments, Ibrahim Chukri, chef du Parti du Travail (branche dite de Sayyida Zaynab, du nom du quartier de son siège, favorable aux islamistes contre la branche socialiste en scission d'Ahmad Migahid qui, elle, a participé aux élections et s'est appropriée le siège de Hadayiq Al-Qubba, les deux branches se disputant la représentation légitime d'un même parti...) ajoute que pour préserver sa marge de manœuvre et de fraude électorale, le gouvernement avait négligé de remettre à jour les listes électorales. Ma'mun al-Hudaybi, côté Frères Musulmans, souligne que «l'augmentation du nombre des circonscriptions a été décidé dans le seul but de décourager les candidats, puisque chacun d'eux doit avoir des représentants dans tous les bureaux de vote de sa circonscription. ${ }^{17}$ Une " Déclaration des Frères Musulmans à la nation au sujet du boycott des élections " reprenait les mêmes arguments en soulignant: "A la suite de la décision de la Haute Cour frappant d'inconstitutionnalité l'Assemblée, la nation s'attendait à une loi électorale qui comporterait les éléments suivants :

\footnotetext{
- un contrôle total des juges sur tous les bureaux de vote (...) et pas seulement sur les sièges généraux qui centralisent les résultats, (...) ce qui aurait pu être réalisé en réduisant le nombre des bureaux de vote et en répartissant les élections sur plusieurs jours ;

- recrutement des juges par le Conseil supérieur de la magistrature, en tant qu'autorité neutre et conformément à l'indépendance du pouvoir judiciaire ;

- vérification de l'identité des électeurs par une pièce d'identité officielle (...) pour couper court à l'utilisation des noms des décédés et des absents.

- renoncement à l'utilisation, comme bureaux de vote, de postes de police et de commissariats.

- rien ne justifie enfin la loi d'urgence, qui a privé le peuple des garanties minimales, a détruit toute vie politique en Égypte et n'a eu pour effet que des troubles sans précédent dans l'histoire de la nation. Il est inconcevable que des élections libres aient lieu sous une telle loi, qui sert de prétexte pour interdire les rassemblements publics et gêner la campagne des candidats.
}

21 Les gouvernants ont annoncé des décrets de lois relatifs à l'amendement de la loi électorale sans prendre en compte les revendications de la nation, et au mépris de la Constitution; et en vertu de ces décrets, la future Assemblée aura le même sort que les deux précédentes. (...) Face à cette désinvolture qui ne peut entraîner que des catastrophes, et par solidarité avec les dirigeants de la nation, les Frères musulmans ont décidé de boycotter les élections prévues pour le mois d'octobre, et de ne pas présenter de candidats. ${ }^{18}$

22 Dans la foulée, le Parti communiste égyptien (clandestin) avait lui aussi décidé de boycotter les élections. Le mot d'ordre « Message à l'électeur égyptien » est lancé sur 
les pages d'Al-Wafd (!) par Nabil Al-Hilali, une des grandes figures du communisme égyptien :

Jusqu'ici, l'électeur tournait le dos aux élections et se murait dans un silence qui en disait plus long que tous les discours. Aujourd'hui, nous attendons de lui un boycott d'un genre nouveau; un boycott actif. (...) Ils le diront que le gouvernement a promis de respecter les libertés et la sécurité des citoyens. (...) Quelle liberté sous la loi d'urgence? (...) Au sein de la gauche égyptienne, des forces ont refusé de camoufler le crime par un masque dérisoire. (...) Elles ont refusé de prendre part au marché électoral ou de le bénir. (...) Ils le diront que le boycott, c'est la fuite face à la confrontation, c'est la coupure avec les masses. (...) Dis leur que c'est la rencontre avec la volonté de la majorité silencieuse, la majorité électorale. Ce n'est que le début de la confrontation avec ceux qui ont bafoué la Constitution. ${ }^{19}$

Outre que ces prises de position constituent un document intéressant pour qui veut y déceler la rhétorique des diverses langues de bois, elles montrent que les partisans du boycott se présentent en porte-parole de l'opposition et, qui plus est, en représentants de la nation toute entière. Ils n'hésiteront pas - on le verra plus loin - à interpréter le taux de participation aux élections comme un succès de leur campagne. Tout cela n'a rien d'extraordinaire dans la mesure où le boycott est qualifié, par l'éditorialiste d'AlCha'b, de "réponse historique »: " Nous ne participerons pas à cette mascarade », titre ce journal.

«C'est la plus importante des décisions de l'opposition depuis l'institution des partis dans les années 70. La décision collective de toutes les forces actives de l'opposition signifie la fin d'une période qui n'a que trop duré. Avec la chute du rideau, le président Moubarak est désormais responsable du blocage des voies pacifiques et légales de la réforme. Désormais la porte est ouverte aux tenants de la violence et de l'extrémisme. $»^{20}$

Pour Al-Wafd, « le parti perdra ses sièges mais il gagnera le respect des citoyens. (...) Les pertes du Wafd et de l'opposition sont minimes par rapport à celle du PND. (...) Il perdra sa crédibilité auprès du citoyen et de l'opinion mondiale. (...) Il monopolisera la scène politique tout seul, comme il l'a toujours fait, mais cette fois sans couverture. $»^{21}$

Toutefois, cette belle unanimité n'a pas été sans accroc. Le Parti des Libéraux, signataire de la déclaration du boycott, s'est illustré par sa position, puisque 22 de ses candidats se sont présentés aux élections; à leur tête... le secrétaire du parti! Une édition spéciale de l'organe du parti présente les candidats ainsi : " 22 candidats se présentent selon les principes du parti malgré le boycott. $»^{22}$ Double exemple d'inconséquence et d'absence de discipline que note l'hebdomadaire Rose al-Yusuf: « Ils ont décidé de se présenter pour prouver le trucage des élections. Si leur secrétaire général n'est pas élu, il s'intitulera «candidat observateur du trucage électoral ! $»^{23}$ C'est d'ailleurs autour de la présence de candidatures indépendantes, parmi les partis du boycott, que va se structurer la réponse aux arguments de l'opposition; les indépendants étant considérés comme le cheval de Troie qui permettra à l'opposition de faire son entrée au parlement tout en appelant au boycott. Le journal AlGumhuriyya réserve ses grands titres à une déclaration du ministre de l'Intérieur selon laquelle « tous les partis et les courants sont représentés ». ${ }^{24}$

Selon Rose Al-Yusuf,

Il est évident que ce sont d'autres intentions qui poussent certains partis d'opposition à boycotter les élections, puisqu'elles y ont déjà participé avec succès dans un contexte beaucoup plus défavorable. (...) Le Wafd a cru pouvoir faire du chantage pour récupérer le prix de son soutien au gouvernement en ce qui 
concerne la crise du Golfe. Il pensait pouvoir gagner des sympathies étrangères, américaines notamment. Par le boycott, le Parti du Travail tente d'éviter une défaite probable, eu égard aux tensions qui le déchirent; d'autant qu'avec un scrutin uninominal, il est loin de pouvoir conserver ses sièges. Les Frères musulmans, eux, ont voulu annoncer tambour battant qu'ils n'attendaient plus rien $\mathrm{du}$ changement par les moyens démocratiques; position qui leur permet de se rapprocher des groupes radicaux. C'est peut-être cela qui explique, chez le RP et la gauche, le refus du boycott; ils ne partagent pas les intentions des Frères, ne peuvent ni ne veulent pratiquer le chantage des wafdistes et, plus important encore, ils ont la possibilité de réintégrer le parlement. ${ }^{25}$

En qualifiant la chute des régimes de l'Est de "démocratie des rues", les observateurs rendaient compte d'un phénomène dans lequel les nouveaux dirigeants étaient choisis par les ovations de la foule après le recul des régimes communistes. Il semble que nos partis d'opposition aient recherché une réaction semblable en décidant le boycott. Ils s'attendaient à ce que les masses les portent vers le pouvoir. (...) La décision du boycott pose une question importante : comment ces partis évaluent-ils leur impact auprès des masses ? (...) Ils se bercent d'illusions, croyant que le gouvernement a pratiqué un trucage pour dissimuler leur importance. Ils sont revenus des tréfonds de l'histoire pour découvrir que les temps avaient changé, et les hommes aussi. (...) C'est en ayant recours à des prétextes anciens qu'ils justifient le boycott. La loi d'urgence est en vigueur depuis 1981 (...) et l'opposition n'est pas sans savoir que dans le contexte actuel, on ne peut se permettre de songer à la supprimer. Par ailleurs le contrôle des juges sur les élections n'a jamais été total, comme le revendique l'opposition, (...) puisque les 7000 juges d'Égypte ne peuvent contrôler 23000 bureaux de vote. Quant au découpage électoral, il concerne simplement les limites géographiques. Il était quelque peu difficile que le gouvernement convoque un congrès public de redécoupage. (...) Les partis du boycott, et avec eux les Frères musulmans, ont constaté que leur opposition au scrutin de liste les avait placés dans une position embarrassante (...), ce même scrutin qui leur avait permis d'obtenir 100 sièges. Avec le retour au scrutin uninominal, chaque parti devait présenter des candidats et des observateurs pour couvrir les 222 circonscriptions, chose qui est impossible compte tenu de leur faible effectif (...) et des coûts de la campagne, qui dépassent leurs possibilités. (...) Il s'agissait d'une forme de chantage pratiqué contre le gouvernement sous la pression de la crise du Golfe pour obtenir des circonscriptions limitées aux seuls candidats du Wafd et de l'alliance islamistes/ Parti du Travail, afin qu'ils gardent le nombre de sièges qu'ils avaient obtenus par le scrutin de liste et qu'ils ne peuvent obtenir par un scrutin uninominal. ${ }^{26}$

Pour Salama Ahmad Salama, de Al-Ahram,

L'argument essentiel de ces partis est qu'ils ne veulent pas servir de décor dans une opération privée de toutes garanties. (...) Le boycott serait une sorte de résistance passive visant à faire pression pour obtenir plus de démocratie. Nous pensons que ce n'est pas là le moyen idéal. (...) Les partisans du boycott n'en sont pas moins des décors, qu'ils le veuillent ou non (...), puisque leur abstention signifie la constitution d'une opposition faible qui ne fera que perpétuer sa présence au parlement sans être en mesure de faire face à la majorité. ${ }^{27}$

L'année dernière, l'équipe des Arab contractors (club de $3^{\text {ème }}$ division. NDLA) a fait la même chose. Les joueurs se sont retirés l'un après l'autre, l'arbitre a annulé le match. (...) Les partis du boycott, eux, se sont retirés d'un seul coup, (...) oubliant qu'il y avait en Égypte des joueurs plus jeunes qui pouvaient mener le match contre l'équipe du National, je veux dire le PND. Autant l'attitude du RP est noble et courageuse, (...) autant est regrettable celle du Wafd, ce défenseur traditionnel de la 
démocratie. (...) Quant au retrait du Parti du Travail, tout Égyptien ne peut que s'en réjouir. Nous souhaitons aussi que son journal disparaisse et que ce parti, prenant son courage à deux mains, annonce sa propre dissolution. (...) Il pourra ainsi se livrer à des occupations qu'il maîtrise mieux que la politique. ${ }^{28}$

Tempête dans une tasse de thé. Si le Wafd n'avait pas, malheureusement, participé au boycott, il n'y aurait pas eu de tempête du tout; juste un petite brise douée sur notre vie partisane faiblarde puisque ce boycott épargne à notre peuple les méfaits d'une participation de la dénommée alliance islamique à la mise au point des politiques publiques et de la législation, les pratiques politiques de ladite alliance s'écartant des intérêts nationaux. (...) Autrefois, le Wafd pouvait se permettre de boycotter les élections en tant que parti de la majorité populaire. (...) Aujourd'hui, dérober le pouvoir au PND, même par des élections libres, est hors de question. Le problème se réduit à quelques députés de plus ou de moins au parlement. ${ }^{29}$

Ce n'est pas le seul son de cloche qui résonne dans la presse officieuse. Ainsi peut-on lire dans le journal Al-Akhbar: «Certaines revendications de l'opposition sont logiques, d'autres le sont moins. Le gouvernement aurait pu prêter l'oreille et rapprocher les adversaires. (...) Le conflit aurait pu être cerné et résolu avec sagesse, mais aucun effort n'a été fait dans ce sens. Le gouvernement n'a pas saisi l'occasion de renforcer ses liens avec une opposition qui lui a accordé son soutien sur la crise du Golfe. ${ }^{30}$ Dans un article intitulé « Des élections propres mais... » Salah al-Din Hafiz, une des prestigieuses signatures d'Al-Ahram, fait le bilan de la situation en ces termes :

A qui faire porter la responsabilité de cette situation? Un PND de plus en plus avide de pouvoir? Une opposition qui a cru pouvoir mettre le gouvernement dans une situation critique et qui a perdu au jeu? (...) Les deux parties sont tout autant responsables l'une que l'autre. Les calculs du parti au pouvoir sont erronés; il a tenu à monopoliser l'élaboration de la loi électorale et refuse de reconnaître, dans l'alternance du pouvoir, le principe même de la démocratie. Les partis du boycott ont eux aussi fait un faux pari : non seulement à cause de la « résistance » du PND mais aussi en raison de leur faiblesse, tant à la base que chez les dirigeants; cela malgré leur expérience, leur presse tapageuse et leur prétention à représenter la .nation. (...) Ces élections tièdes feront pourtant partie des quelques rares élections propres pour la simple raison qu'il n'y aura guère besoin de les trafiquer. (...) Je désespère d'aller plus avant dans l'idée que j'avais proposée : constituer un comité restreint de personnalités dignes de confiance qui se prononcerait sur la régularité des élections. ${ }^{31}$

La gauche au Parlement

Le retour de l'opposition de gauche à l'Assemblée du Peuple après 11 années d'absence s'est-il opéré à cause de l'absence des autres forces d'opposition ou malgré elle? S'agirait-il d'un comportement de «vote utile» destiné à pénaliser le parti au pouvoir ? Le gouvernement aurait-il décidé de 'marginaliser les partisans du boycott en donnant la preuve du sérieux de ses engagements démocratiques et en posant en même temps les bases d'une nouvelle règle du jeu - parlementaire cette fois-ci - entre pouvoir et opposition de gauche? S'agirait-il, comme le suggèrent quelques interprétations d'Al-Cha'b, du "marché » conclu entre le Rassemblement progressiste et le pouvoir, en vertu duquel quelques circonscriptions seraient laissées à la gauche en y mettant des candidats fantoches du PND ? ${ }^{32}$ Faut-il y voir tout simplement un indicateur du niveau d'immersion et de popularité des figures de la gauche dans le cadre d'élections libres? Les réponses ne s'excluent pas, mais les données de la bataille électorale ne 
permettent pas de trancher. La question est évoquée par un sympathisant du RP dans les pages d'Al-Ahali :

Toutes les composantes de la gauche ne peuvent que se féliciter des succès réalisés. Mais il faut prêter l'oreille aux rumeurs selon lesquelles le gouvernement aurait "permis» à la gauche de passer au parlement, ce qui pour moi ne signifie aucunement une quelconque complicité entre la gauche et le pouvoir, ni qu'elle aurait réalisé un succès non mérité. Cela veut dire que le pouvoir a ressenti que la présence de la gauche était nécessaire et n'a pas eu recours aux méthodes habituelles. Est-ce pour des raisons de rapprochement idéologique avec la gauche? Certainement pas. (...) Les élections expriment-elles un tournant populaire à gauche? Le succès limité ne permet pas de le conclure. (...) L'orientation est contraire aux courants religieux, dont certains tentent de s'imposer par les armes. On ne peut nier également que l'élection de certaines grandes figures de la gauche est due davantage aux personnes qu'à l'idéologie, à commencer par Khalid Muhyi-lDin. (...) En dépit du fait qu'il s'agit d'un régime de droite, le régime actuel pressent le danger que représente la droite religieuse et tente de jouer le jeu inverse de son prédécesseur. Hier on donnait le feu vert aux groupes religieux pour faire face à la gauche; aujourd'hui la menace vient de ceux qui parlent au nom de la religion. Ouvrons donc les médias à la gauche, et laissons-leur des bulletins de vote. (...) Les alliances politiques changent et les ennemis d'hier peuvent devenir les amis d'aujourd'hui. La gauche se doit de saisir de cette possibilité pour raffermir ses liens avec les masses et mieux se faire connaître ; c'est un droit indéniable. (...) J'espère que les élus auront la vigilance nécessaire, pour diriger leur énergie vers le dialogue et non vers la confrontation. (...) La gauche ne doit pas oublier qu'elle se place sur le terrain d'un jeu dont les règles ne sont ni " propres ", ni clairement définies. ${ }^{33}$

La conjoncture en vertu de laquelle nassériens et députés du RP se retrouvent sous la coupole du parlement au moment même ou le Wafd et la coalition Parti du Travail/ Frères Musulmans s'en absentent, cette inversion des places et des rôles entre les forces politiques (qu'on aurait pu interpréter comme une cristallisation des conflits sociaux dans le cas d'un fonctionnement «normal» de la représentativité) est trop lourde de sens pour qu'on puisse écarter la question ou la mettre sur le compte du hasard, quand bien même elle serait la résultante d'élections neutres. Et dire que la présence de l'opposition de gauche au sein du parlement est quasi symbolique ne fait que souligner son importance « symbolique »...

Tout en partageant les réserves des partis du boycott à l'égard des modalités des élections, le RP en tire des conclusions et une attitude différentes et se retrouve dans une position défensive, justifiant sa participation. Ces justifications sont de deux ordres; liées d'une part au contexte régional et national - la crise du Golfe plus particulièrement - et d'autre part aux modalités même de la bataille électorale, puisque le scrutin uninominal, selon le RP, limite la portée du boycott et laisse le champ ouvert aux candidats indépendants. "Pouvons-nous nous retirer dans une telle situation?", écrit Ahmad Saïd Ahmad dans le journal Al-Ahali, porte-parole du RP.

La gravité de la situation actuelle est une des raisons pour lesquelles le RP insiste pour participer à la bataille électorale. Un parti politique peut-il se permettre de se couper des masses alors qu'en ce moment se décident des questions de la plus haute importance, et que le RP a une position bien définie à l'égard de ce qui se passe dans la région? .En appelant les masses à voter pour ses candidats, le RP rappelle ses positions claires. (...) Il y a tout d'abord le problème de la guerre et de la paix. Le RP a adopté une position sans ambiguïté en faveur d'un règlement pacifique. (...) Il est nécessaire (te trouver la formule - difficile certes mais indispensable et la seule en mesure d'éviter à cette région une catastrophe - qui permette un retrait irakien du Koweït par des moyens pacifiques et non par une solution militaire. C'est un 
objectif essentiel qu'on ne peut réaliser en laissant la décision à des partenaires étrangers qui décideraient en fonction de leurs intérêts. Il y a également l'avenir de la nation arabe. (...) Si la crise est résolue par la guerre, il n'y aura plus d'entité arabe unifiée. (...) Dire que la guerre est inévitable, c'est admettre la fin du système arabe. Pour que l'intervention étrangère dans le Golfe soit légitime, il faudrait qu'elle adopte à l'égard des Arabes et d'Israël un seul poids, une seule mesure, et qu'elle fasse le lien entre le Golfe et la Palestine. (...) Il nous faut évoquer par ailleurs l'opération en cours qui consiste à éponger la dette égyptienne ; opération que nous appuyons bien évidement, mais nous tenons à ce que le peuple égyptien sache quel en est le coût politique. (...) Il y a là des problèmes qu'il faut aborder avec fermeté. Je n'imagine pas que les partis d'opposition en Égypte puissent réclamer le moindre rôle s'ils abdiquent, dans ces circonstances, leurs responsabilités politiques face à dés questions qui touchent l'avenir. ${ }^{34}$

À la question «Pourquoi le RP participe-t-il à des élections boycottées par l'opposition? » le secrétaire du comité central du donne cette réponse :

Notre parti soutient la lutte pour que soient garanties des élections neutres et appelle à l'unité de toutes les forces pour les obtenir. (...) Le boycott ne peut faire l'objet d'un consensus populaire propre à réaliser ces objectifs, notamment par le biais d'un scrutin uninominal qui permet à des dizaines de candidats de se présenter. Quand le scrutin de liste était en vigueur, et tandis que le boycott entraînait l'absence totale de toutes les forces d'opposition, nous les avons appelées à boycotter; mais elles ont refusé. Voici qu'aujourd'hui ce sont elles qui appellent au boycott. (...) Le boycott en vue d'une réforme globale ne pourra pas réaliser ces objectifs. (...) Il ne sera plus possible, dès lors, de convaincre les masses du bienfondé du changement par les votes démocratiques. (...) Ne restera comme issue que le changement par la violence. (...) Il est certain que le pouvoir reste entièrement responsable de la montée de la violence et de l'extrémisme religieux. (...) Mais nous reconnaissons nos responsabilités envers ce peuple et la nécessité d'y couper court. (...) Les élections sont en elles-mêmes une possibilité de contact avec les masses qui permet d'induire une vitalité politique et de faire face aux tenants de l'extrémisme. (...) Le boycott par le haut est assurément un geste politique; mais il est loin de signifier un boycott des masses.

Répondant aux propos du Parti du Travail selon lesquels là participation du RP est le prix d'un marché conclu entre la gauche et le pouvoir, le secrétaire du comité central rejette ces accusations : "Comment le Parti du Travail peut-il soutenir de tels propos, alors que tout le monde sait ce qu'il en a été des marchés qu'il a conclus avec le président Sadate, à commencer par celui dont le prix a été sa constitution même?» Commentaire du journal : "II semble que le RP ait décidé depuis une assez longue période de déclencher une bataille contre les courants religieux et les Frères Musulmans. $»^{35}$

C'est encore dans Al-Ahram qu'on trouve - une fois n'est pas coutume - un panégyrique sur le chef du RP : «Hommage à Khalid Muhyi-1-Din qui a refusé le boycott de façade. Hommage à lui car il a guidé son parti vers le juste choix, défiant ceux qui voulaient le tirer vers l'arrière. Grâce à Dieu, il l'a remporté haut la main et aura désormais le droit d'être à la tête d'une opposition libre et honnête dans cette Assemblée qui s'annonce comme l'une des plus solides, choisie par le peuple. Nous espérons qu'au deuxième tour, le RP puisse obtenir encore d'avantages de sièges. ${ }^{36}$ II faut croire en revanche que l'étiquette nassérienne dérange au point de ne pas être citée, alors que l'argument principal du gouvernement, repris par le ministre de l'Intérieur, est que «toutes » les forces politiques participent aux élections. La presse officieuse n'a pas évoqué les candidatures nassériennes ni l'élection d'élus nassériens à l'exception de Dia' al-Din 
Dawud, grande figure de l'ancienne génération nassérienne. Elles ont été présentées par le journal du RP en ces termes: "C'est en partant des positions de principe relatives à la nécessité de développer les liens avec les masses, et pour briser les barrières que certains tentent de dresser autour d'eux, les considérant comme des forces non légitimes, que plusieurs candidats nassériens ont décidé de se présenter aux élections. $\aleph^{37}$ Le paradoxe est qu'au moment même où les conditions de "visibilité " politique semblent favorables au courant nassérien - force politique qui, bien que privée de parti, a remporté des sièges au Parlement, ce qui témoigne de son existence et conforte ses revendications relatives à la constitution d'un parti - le mouvement des nassériens semble moins que jamais préparé à ces nouvelles dispositions. Traversé par des clivages internes d'une part, menacé d'autre part dans sa cohérence idéologique par la généralisation du réfèrent identitaire/religieux, que la crise du Golfe ne manquera pas d'exacerber, le courant nassérien semble confronté à des choix décisifs pour son existence même.

L'épreuve des nouveaux partis

39 La couverture médiatique qu'ils ont reçue était à la mesure de leur envergure, c'est-àdire fort réduite. A l'exception de la campagne quasi quotidienne menée par le «billettiste " 'Abd al-Salam Dawud, membre fondateur des Verts, dans sa rubrique du journal Al-Akhbar et de la parution, quelques semaines à peine avant les élections, de Misr al-Fatat, organe du parti du même nom, on ne trouve que fort peu d'éléments sur ces organisations ${ }^{38}$.

Si le RP a estimé que les conditions étaient favorables au retour de ses députés au parlement, pour les nouveaux partis il s'agissait d'une grande première; leur «sortie nationale ", si l'on peut dire. Mais n'était-elle pas quelque peu prématurée, puisque c'est six mois avant les élections que ces partis - dont les dirigeants sont peu connus du public - ont vu le jour?

41 Le Parti des Verts - muni de slogans dont le moins qu'on puisse dire est qu'ils sont peu « accrocheurs » dans une société non sensibilisée aux problèmes écologiques et dans un paysage politique où les mouvements « à cause unique » recueillent peu d'adhésion - a présenté 19 candidats et n'a pu obtenir aucun siège. Il s'agissait pour une large part d'universitaires peu rompus aux techniques du jeu électoral, bardés de diplômes et de prix internationaux qui n'ont visiblement pas impressionné l'électeur.

Notre décision de participer aux élections, dit un de leur responsables, repose sur deux ordres de considérations. Il s'agit en premier lieu de promouvoir la notion d'environnement. Nous croyons par ailleurs que la fonction première d'un parti est d'approfondir les notions de liberté et de participation aux élections parmi les masses. (...) Les Verts n'entrent pas sur la scène politique comme un parti d'opposition, même s'ils ont été perçus et classés comme tels. Cela ne figure pas sur notre programme ni dans nos intentions. Nous pensons que tous les partis devraient se joindre à nous dans la mesure où nous appelons à la protection de l'environnement. C'est en partant de ces considérations, et indépendamment des résultats de la bataille électorale, que nous avons décidé de participer aux élections. 39

42 Le témoignage de l'un d'eux, inventeur d'un produit pour la bonification des terrains agricoles, illustre bien leur position :

Avant que je ne fasse les premiers pas, les professionnels des élections avaient déjà installé leurs banderoles sur les places centrales, organisé la préparation des meetings dans les cafés, mosquées, duos, monopolisé toutes les agences de location de matériel, tous les calligraphes... (...) Ma campagne, je l'avais concentrée sur le 
prix pour l'environnement accordé par l'ONU. (...) J'ai découvert qu'il s'agissait d'autre chose (...) : entrer dans les cafés, payer la tournée aux clients, commencer à parier comme le font les autres... Je n'en ai pas le courage. ${ }^{40}$

Toujours à propos des Verts, on peut lire sous la plume de 'Abd al-Salam Dawud : (sic) et simplifiée (sic) du programme des Verts (...), dont le but est de faire connaître à l'électeur les objectifs de ce parti naissant. Cela est nécessaire dans la mesure où ces objectifs sont nouveaux pour le citoyen de culture moyenne qui n'a jamais entendu parier du trou dans l'ozone, de la couche de gaz carbonique, de la montée du niveau des mers ou autres graves dangers qui menacent non seulement notre pays mais l'univers entier. Cela ne veut pas dire que les Verts planent dans les hauteurs de la science, loin des préoccupations de l'homme de la rue; bien au contraire, ils ont leur opinion - modérée et scientifique - sur beaucoup de problèmes quotidiens. Ils vous invitent à jeter un œil sur ce programme, non parce qu'ils sont à là recherche d'un succès électoral - leurs 19 candidats ne formeraient pas une majorité même s'ils étaient tous élus - mais parce qu'ils souhaitent se faire entendre sous la coupole de l'Assemblée. ${ }^{41}$

44 A la veille du deuxième tour, le journal Al-Ahram fait le bilan de ce qu'il identifie comme «les partis de la marge»:

L'ombre de la défaite est tombée sur les partis de la marge après l'échec de tous leurs candidats. Le premier effet de cette défaite est la démission de Hasan Ragab, président des Verts, pour « raisons de santé » et « raisons de travail »; Hasan Ragab achève en ce moment son encyclopédie sur l'université d'Alexandrie. S'il a tenu à juxtaposer ces deux arguments, c'est que la réalité est ailleurs. Sa démission est liée à des facteurs internes à l'organisation qu'il représente. M. Ragab était partisan de ne pas se présenter aux élections dans la mesure où le parti, avec six mois d'existence à peine, n'avait pas constitué de base populaire. Il a été obligé de renoncer à cette position sous la pression de certains éléments de son parti. Misr alFatat (16 candidats) a réussi pour sa part à adjoindre à ses rangs, entre le premier et le deuxième tour, un candidat indépendant en ballottage pour avoir un représentant au sein du parlement (te candidat en question n'a pas été élu au deuxième tour. NDLR) Quant à 'Abd al-Mun'im Turk, chef du Parti al-Ittihadi, il a préféré fermer le siège de l'organisation à Alexandrie et a refusé de commenter les résultats de ces élections auxquelles il avait présenté trois candidats, tous perdants. ${ }^{42}$

Le parti «familial » de Ahmad al-Sabbahi, al-Umma, qui n'a pas réussi à remporter de sièges, est lui aussi assimilé aux partis de la marge même s'il a sept ans d'existence. "Ces élections - estime Al-Sabbahi quelques jours avant le déroulement du scrutin sont une chance inestimable pour les partis de la marge ; je pense que l'opposition aura toutes ses chances. $»^{43}$ Pour Al-Ahram, les résultats du Parti al-Umma ne sont pas surprenants : «II a échoué aux cinq élections auxquelles il a participé pendant ses sept ans d'existence. Cela n'empêche pas Al-Sabbahi de rejeter la responsabilité de son échec sur le PND. $»^{44}$

La nébuleuse des indépendants

C'est l'événement principal de ces élections (60\% des candidatures), doublement intéressant et par ses composantes politiques et parce que c'est à travers ses composantes sociologiques qu'on peut lire une des "faces cachées» de la société égyptienne. Elle se compose de plusieurs éléments. En premier lieu, certains membres du PND qui n'ont pas été choisis par leur parti ; l'hebdomadaire Rose al-Yusuf estime que " désireux de renouveler sa classe politique et soucieux en même temps de préserver sa clientèle, le PND a encouragé certains de ses membres à se présenter comme indépendants. $»^{45}$ On le verra plus loin, les candidatures individuelles des 789 membres 
du PND - contre 444 candidats du parti, quasiment le double - n'ont pas manqué de susciter quelques conflits sur le terrain, reflet des conflits de pouvoir au sein de la sphère politique de l'organisation.

Viennent ensuite les candidatures des membres des partis du boycott, qui ont jugé opportun de se présenter en faisant fi des décisions de leurs organisations ; les chiffres donnés par le ministre de l'Intérieur sont de 81 candidats pour le Wafd, 71 pour le Parti du Travail, 34 pour les Libéraux. A ceux-là, Al-Ahram ajoute les "vrais indépendants ", qui n'ont de liens avec aucun parti ${ }^{46}$. Il nous faut compter parmi ceux-là - même si leurs identités n'ont pas été clairement énoncées - les candidats des forces politiques privées de partis, des nassériens essentiellement et quelques figures des courants islamiques. Viennent enfin les nouvelles recrues, inconnus au sein de la classe politique mais peutêtre pas dans leur quartiers; ces "monsieur-tout-le-monde ", dont on connaît peu de choses et qui pourtant sont de précieux révélateurs des centres de pouvoir à l'intérieur de la société égyptienne, et peut-être de rapports au politique, tant individuels que collectifs/communautaires, qui restent à découvrir. Ce n'est pas ce qu'en pense Mustafa Kamal Murad, chef des libéraux: "II s'agit le plus souvent de mégalomanes, plus intéressés par leur propre publicité que par l'activisme politique et le travail social. La plupart d'entre eux s'appuient sur les solidarités communautaire ('açabiyya) et les grandes familles. $»^{47}$ Savoir si les $"$ autres » candidats sont élus en fonction de critères « autres » est une question qu'on verra plus loin.

Indépendamment des résultats - 83 sièges dont 14 pour le Wafd, 8 pour le Travail et 1 pour les Libéraux selon les chiffres du ministère de l'Intérieur, qui persiste à les identifier selon leurs partis ${ }^{48}$-, le fait de poser sa candidature - tout comme le fait de voter, surtout lorsqu'il s'agit d'une pratique peu répandue ! - est en soi chargé de sens : qu'il émane d' « indépendants-indépendants ", et c'est un pan caché de la société qui se dévoile. Venant $d$ « indépendants-partisans ", il nous en dit long sur la discipline et le fonctionnement interne de ces partis politiques qui, finalement, ne seraient pas aussi étrangers qu'on le dit au fonctionnement de la société toute entière. C'est ce dernier aspect qu'a retenu la presse. Interrogé par le journal Al-Gumhuriyya, Mustafa Kamal AlSayyid, politologue, explique le " phénomène des indépendants » en ces termes :

II y a tout d'abord la faiblesse de l'identification partisane. Nombreux sont les candidats qui se sont présentés contre les décisions de leurs partis, notamment au sein du PND. (...) Les menaces d'exclusion ne sont pas prises au sérieux. Une grande partie des citoyens refuse par ailleurs l'activité politique collective et préfère la pratiquer sans devoir se lier à un parti. Cette attitude renvoie à des traits culturels égyptiens, plus particulièrement ceux des classes moyennes : individualisme absolu, refus de coopérer, refus de s'astreindre aux règles d'un travail collectif. Cependant, on constate une tendance analogue dans les sociétés avancées, où ce que les partis politiques perdent de crédit est récupéré par les mouvements sociaux (pacifistes, féministes, écologistes...). Il ne semble pas qu'il y ait en Égypte des mouvements sociaux qui puissent tirer profit de la faible popularité des partis. Par ailleurs, ceuxci ne sont pas en mesure d'attirer toutes les forces politiques qui jouent un rôle en dehors du cadre des partis et n'ont pas droit à l'expression. Des partis légitimes sans base populaire et des forces politiques présentes dans la rue et privées de légitimité. ${ }^{49}$

L'impact du succès des candidats indépendants a été perçu de différentes manières. Selon l'éditorialiste d'Al-Ahram,

L'apparition de personnalités indépendantes que l'on peut considérer comme dignes de respect et qui jouissent de popularité parmi les masses, soit pour leurs prises de position par le passé, soit pour leur réputation ou leur statut social ou 
scientifique, (...) relève le niveau de la participation et souligne que ces personnalités sont liées au régime, indépendamment de leurs légitimes divergences d'opinion. (...) Le boycott que nous n'aurions pas souhaité a ouvert la voie à des éléments plus jeunes et des figures nouvelles. Par conséquent, le cercle de la participation s'est élargi et les nouvelles générations manifestent plus d'intérêt pour l'activité politique. ${ }^{50}$

Cet optimisme n'est pas partagé par tout le monde. Pour les partisans du boycott, il n'est pas envisageable que ces députés jouent le rôle de l'opposition. Pour Ma'mun AlHudaybi, "pluralisme démocratique signifie pluralité des partis qui, à leur tour, représentent des forces données. (...) Un individu isolé ne peut pas grand chose. L'existence d'un grand nombre d'indépendants est un phénomène néfaste. (...) L'opposition suppose un parti et des idées; celui qui n'en a pas ne peut la représenter. $»^{51}$ Un membre du Wafd ajoute :

C'est bien la pire des Assemblées que nous ayons connues. D'ailleurs elle ne durera pas longtemps à cause de l'absence de l'opposition. (...) Il est exclu que celle-ci puisse être représentée par les indépendants. (...) Un grand nombre d'entre eux appartient au PND; le seul but de l'opération était de monter une "comédie électorale ». Ces indépendants sont dépourvus de programmes auxquels se référer durant les débats, et leur dépendance vis-à-vis de leur circonscription les cantonne dans une vision étroite. ${ }^{52}$

Que les élus indépendants soient perçus comme les futurs suppôts du pouvoir ou les acteurs d'une comédie mise en scène par le PND, le fait est qu'ils se trouvent coincés entre une majorité écrasante et un RP détenteur de 6 sièges, même si cela fait de son président Khalid Muhyi-l-Din le chef de l'opposition. Nombre d'entre eux appartenant au PND ou aux organisations de l'opposition, il était inévitable que soit posée la question de cette appartenance partisane une fois la mission accomplie, et du sort qui serait fait aux décisions des uns et des autres d'exclure tout membre qui aurait transgressé le mot d'ordre de boycott. La réponse est donnée par Al-Ahram : «Je vous ai eus : c'est le nom du parti des indépendants qui ont crié haut et tort leur opposition à tous les partis, le PND en tête et qui, aussitôt entrés au parlement, se sont précipités vers ledit PND, disant à leurs électeurs : je vous ai eus... " ${ }^{53}$

Un candidat indépendant et élu en tant que tel a-t-il le droit de modifier son appartenance et d'intégrer un parti? Question d'Al-Ahram au politologue Ali al-Din Hilal: "Ceux des élus qui n'avaient pas d'appartenance politique avant les élections doivent sauvegarder leur indépendance pour constituer le noyau de futurs partis politiques éventuels. » Pour un autre politologue, changer d'appartenance est un crime commis contre la morale et vis à vis des électeurs. (...) Tant qu'aucune loi n'interdira cette pratique, l'appartenance partisane en Égypte restera sans valeur. Yahya Al-Gamal, professeur de droit constitutionnel et membre du PND, donne cette réponse désabusée : "Rien, dans la loi ni les usages, n'interdit à l'élu indépendant de se joindre à un parti, que celui-ci soit présent ou non au parlement. (...) D'un point de vue politique, il nous faut voir la question de plus près et le constat est douloureux. Les batailles électorales se décident-elles chez nous en fonction de l'appartenance politique? Avec le scrutin uninominal, le choix est déterminé en fonction de deux critères, la 'açabiyya et les « services personnels »... Tout cela n'a rien à voir ni avec les partis, ni avec la politique ni même avec les élections, sans parler de la volonté populaire. » Le PND avait lui aussi menacé d'exclure les récalcitrants. L'un de ses responsables déclare: «Je ne peux interdire l'accès au parti à ceux qui veulent y rester. Je suis certain que les membres qui se sont présentés en marge de nos candidatures tiennent à leur appartenance au parti 
et à ses principes. Je ne peux non plus leur interdire le droit de se présenter. - Qu'en serait-il s'ils refusaient de se joindre au comité parlementaire du PND ? - Je vous assure qu'il n'en est rien. $»^{54}$

53 Yusuf Wali, secrétaire général du PND, répond à la même question : «Cela dépendra des députés eux-mêmes. S'ils s'inscrivent sur les listes du parlement sous l'étiquette PND, nous allons reconsidérer leur position. Les autres sont libres également et nous prendrons à leur égard les dispositions qui s'imposent. » Les libéraux, pour leur part, qui n'ont de sièges au parlement que celui que leur attribuent les décomptes du ministère de l'Intérieur, annoncent: "Nous avons exclu les membres qui n'ont pas respecté le boycott et nous ne reviendrons pas sur cette position. $»^{55}$ était, lui, favorable à la participation, "une quarantaine de nos membres se sont présentés, qui sont loin d'être de grandes figures. Ils avaient peu d'impact à l'intérieur du parti et leur absence ne nous affecte en rien. S'agissant des élus, il n'y en a que quatre. (...) Le comité supérieur du parti prendra les décisions qui s'imposent. ${ }^{56}$

A propos de la manière selon laquelle l'opposition va s'organiser à l'Assemblée, le journal Misr Al-Fatat évoque une lutte sourde qui oppose d'une part Khalid Muhyi-lDin, chef de l'opposition en coalition avec ses députés du RP, les indépendants de gauche et les nassériens, tous regroupés dans un front d'opposition élargi, et EIwi Hafez, député en rupture avec le Wafd qui envisage de constituer un Parti des indépendants du nom de Al-Taççadi (la confrontation). ${ }^{57}$

Les brebis égarées, les brebis galeuses finiront-elles par regagner le bercail ? Elus et perdants auront-ils droit au même traitement ? Faute de pouvoir en dire plus long dans cet imbroglio politique, et pour essayer d'aller plus avant, là ou se trouvent les enjeux de cette bataille électorale, du moins quelques uns (ne pas oublier ici les gratifications matérielles et symboliques auxquelles donne droit le titre d'élu, sans parier de celles qu'il "permet » en termes de dons et contre-dons, bien au-delà de la fonction de représentant de la nation), il faudrait peut-être mentionner - simplement à titre d'hypothèse - ces propos d'un membre du bureau exécutif des Verts :

Je pense que les indépendants seront amenés à jouer un rôle réel pour une raison importante : c'est qu'ils appartiennent tous au capitalisme. Ils croient au marché libre, cela correspond à la tendance mondiale et plus particulièrement aux besoins de l'économie égyptienne; il est donc normal qu'ils représentent un poids économique et politique. (...) Ils auront leur point de vue sur la solution des problèmes économiques et pousseront vers l'économie libre. C'est pour cette raison qu'ils ont obtenu une grande part des sièges de l'Assemblée..$^{58}$

Ahmad Bahgat, dans sa rubrique quotidienne à Al-Ahram, propose la même lecture :

II est certain que les indépendants formeront un front d'opposition indépendamment des partis. Plus autonome certes, mais plus fluide aussi et tacitement manipulable par le PND. (...) A considérer les sommes énormes dépensées pour la campagne électorale, il s'agit à n'en pas douter de capitalistes. (...) Ce ne sont pas des partisans de politiques de demi-mesure (...) et leur présence au parlement va favoriser la transition vers l'économie libre. Le capitalisme égyptien a enfin réalisé l'intérêt qu'il a à prendre part à la sphère législative et qu'il ne peut grand chose en dehors de cette sphère..$^{59}$

58 A l'heure où est lancé le programme de libéralisation de l'économie égyptienne, dit "programme des mille jours ", reste à savoir si c'est à travers la nouvelle Assemblée 
que se feront entendre les voix (ou les échos) tant des bénéficiaires de la libéralisation que celtes de ses victimes...

Des exclus d'un autre " genre "

Après les exclus du politique, les exclus des partis et «ceux qui se sont exclus euxmêmes", selon la logomachie en vigueur dans ces cas, viennent les exclus du genre féminin. Sur les 444 candidatures du PND, elles étaient quatre candidates en tout et pour tout, fait qui n'a pas été passé sous silence par les plumes féminines (féministes) notamment. Rappelons ici qu'une disposition de la loi électorale réservant 30 sièges aux "élues» avait été supprimée par la Haute Cour constitutionnelle pour cause d'inconstitutionnalité.

Par un simple calcul, écrit Husni Chah dans Al-Akhbar, on constate que la proportion des femmes présentées par le parti au pouvoir est de $0,09 \%$. Il est clair que le plus important des partis en Égypte aura ainsi décidé de tirer le tapis sous les pieds des femmes en ce qui concerne la vie parlementaire, pour 5 ans au moins ; la proportion des femmes est quasi inexistante, surtout si on met à part. Mme Amal 'Uthman, ministre des Affaires sociales, choisie en fonction de son portefeuille ministériel. De deux choses l'une : ou c'est la direction du parti, responsable du choix des candidats ensuite soumis au président de la République, qui ne croit pas au rôle politique de la femme (...) et l'estime indigne de représenter le peuple; ou bien ce sont les cadres féminins à l'intérieur du parti - elles sont plusieurs centaines - qui ont donné les preuves d'un lamentable échec. ${ }^{60}$

Pour Fu'ad «Pacha » Sirag al-Din, chef du Wafd, interviewé par une journaliste de l'hebdomadaire Sabah al-Khayr, « le fait que les femmes aient atteint moins de 1\% est une régression, un retour en arrière. Il y a quelques années, elles avaient 30 sièges au moins. Beaucoup d'Égyptiennes ont un niveau de culture politique élevé et je ne sais pourquoi le PND les a ignorées. Certaines ont réussi à faire abstraction de leur sexe (! NDLA) et ont pris des positions courageuses au sein du parlement. Une femme ne peut pas exercer de fonction dans la magistrature pour ne pas sympathiser avec l'accusé ; mais celles d'entre elles qui étaient au sein de l'opposition parlementaire n'ont pas fait du sentiment en ce qui concerne le parti au pouvoir. » Ibrahim Chukri, chef du Parti du Travail, pense pour sa part que « pour de nombreuses raisons ancrées dans la société, le scrutin uninominal n'offre pas de chances réelles aux femmes. Il faut que les partis politiques tentent de remédier à cette situation pour que les femmes soient représentées dans toutes les instances élues. » Même opinion pour Rifat Al-Sa'id, du RP, qui ajoute :

La cause de la femme est en elle-même une cause politique, et non une question spécifique aux femmes. Elles auraient mieux fait, d'ailleurs, de concentrer leurs efforts sur cette cause. Dans notre lutte contre l'obscurantisme et contre ceux qui essaient de faire marche arrière, un élément principal est la libération de la femme et la défense de ses droits. Le problème des femmes est qu'elles n'ont pas pu faire entendre leur voix dans les assemblées précédentes. Peut-être ont-elles été terrorisées par ceux qui appellent au retour en arrière; la question de la femme concerne tant les femmes que les hommes. Jusqu'ici, cette question n'a pas été considérée comme prioritaire par les « députées » elles-mêmes ; j'espère qu'elles la prendront en compte. ${ }^{61}$

L'absence des femmes fait partie, d'après le conseiller Muhammad Sayyid al-Achmawi, de la passivité qui caractérise de façon générale les Égyptiens :

II n'existe pas de participation politique des femmes au vrai sens du terme. Elles ont été habituées à ce que le pouvoir leur accorde leurs droits (...) et ne cherchent plus à organiser le mouvement qui leur permettrait de les défendre. (...) Il ne faut pas 
oublier également l'impact des pays arabes et islamiques voisins, pour lesquels la place d'une femme est au foyer: elle ne peut en aucun cas participer à la vie publique, politique ou autre..$^{62}$ voix du ministre d'État aux Affaires de l'Assemblée et du Conseil consultatif, répond en invoquant l'âpreté de la bataille électorale dans un scrutin uninominal : « Dans ce mode de scrutin, le parti n'a pas de contrôle sur ses membres. Tout citoyen peut se présenter aux élections. L'opération est beaucoup plus complexe et le scrutin uninominal exige des efforts inouïs. Les candidats se renvoient des critiques et cela va quelquefois audelà des critiques, ce que nous voulions éviter. Nous aurions voulu présenter 30 candidates mais la femme ne peut, de par sa nature même, affronter l'agressivité des concurrents ni fournir l'effort physique qu'exige ce mode de scrutin. Les femmes sont trop chères à nos cœurs pour que nous les exposions à ces risques. » Réponse de la célèbre Aycha Ratib, ancien ministre des Affaires sociales et ex-ambassadrice: « Ce n'est pas ainsi que l'on rend hommage à la femme égyptienne. (...) L'argument présenté est un faux prétexte pour justifier l'exclusion de la moitié de la société. On peut envisager de réserver quelques circonscriptions aux candidates et le parti devrait les soutenir comme il le fait pour ses candidats. $»^{63}$

$\mathrm{La}$ « réponse à la réponse » est présentée en ces termes :

Elle s'est enfin réveillée une fois les préparatifs terminés et a découvert - oh malheur! - que les politiciens et les partis l'avaient trompée. Ils ne l'ont pas inscrite sur les listes de candidatures, ne l'ont pas guidée vers les circonscriptions ou le succès est le plus garanti pour qu'elle soit certaine de pouvoir représenter la moitié de la société, voire le vingtième ou le quart faute de mieux. Ce n'est qu'une fois passé le délai de dépôt des candidatures que la femme égyptienne a manifesté une grande activité, critiquant parti de la majorité. (...) Je soutiens personnellement le droit de la femme à participer à la vie politique, sociale et partisane, mais c'est elle qui a causé sa propre défaite. Elle a renoncé à ses droits, à son rôle politique et social, s'est contentée des miettes laissées par les hommes et s'est laissée reléguer à une fonction purement décorative dans les réunions, les congrès et les colloques. (...) J'ai écris ailleurs que la femme égyptiennes démissionné dès le jour ou elle a capitulé face aux modifications du statut personnel, et qu'elle a accepté la défaite face aux courants de la réaction. (...) Il est certain que les partis politiques n'auraient pas donné la priorité aux femmes de leur propre gré, notamment avec un scrutin uninominal dans lequel la personnalité du candidat est un facteur décisif. Si les partis politiques se caractérisent par la faiblesse de leurs cadres masculins, qu'en serait-il alors pour les cadres féminins qui, dès le départ, n'ont pas marqué leur présence? Quant aux associations, à la presse et aux grandes figures féminines, elles ne se sont réveillées qu'après qu'on a tiré le tapis sous leurs pieds. Elles en sont les seules responsables. ${ }^{64}$

II n'est pas sans intérêt de rappeler ici que sur une vingtaine de candidates (dont plusieurs membres du PND non désignées par le Parti) 7 ont été élues. Elues non grâce au PND mais en raison de leur popularité et des activités qu'elles mènent dans leurs circonscriptions, comme tient à le rappeler la journaliste d'Al-Akhbar en interviewant les élues: «Les sept candidates ont remporté une grande victoire sur les hommes, et cela malgré l'attitude du PND. (...) En réalité, le parti a été davantage un fardeau qu'un soutien. C'est la grande popularité des candidates qui a soutenu le parti et leur a permis de l'emporter. L'attitude du PND montre qu'il avait une vision courte des choses et qu'il n'a pas fait les bons calculs. Sa position vis-à-vis des candidatures féminines a laissé un impact négatif dans les milieux proches du mouvement des femmes. $»^{65}$ 
totale d'élus et de candidats chrétiens. Pudeur qui caractérise le débat sur les questions
confessionnelles de manière générale, et débat d'autant plus ténu, voire " honteux ",
dans la mesure où ce n'est plus l'extrémisme/confessionnalisme des uns qui est mis en cause, mais bien les dispositions du parti au pouvoir, l'ensemble des partis présents dans la compétition électorale et le rapport d'une communauté religieuse à la politique. L'ensemble de ces éléments se retrouve dans le débat limité, lancé cette fois-ci par le journal du Wafd - tradition libérale exige - et relancé si on peut dire ainsi par les réactions intercommunautaires. Pour Al-Wafd, la sous-représentation des coptes est comprise dans le cadre plus général de sous-représentation des minorités :

II suffit de jeter un coup d'œil aux candidatures du PND pour se rendre compte du peu «d'intuition politique» de ce parti et de l'inconscience totale qui dicte ses choix et ses thèses politiques. Un exemple flagrant en est l'omission de représentation réelle et équilibrée des minorités. Sur 444 candidats, le part a proposé deux coptes alors qu'ils sont 3 millions, selon le recensement de 1989. Pour 2 millions de femmes ils ont proposé 4 candidates, alors qu'elles étaient 13 dans l'Assemblée précédente. Les Nubiens, qui représentent une communauté égyptienne spécifique par ses problèmes, son environnement et son dialecte, ont été totalement ignorés. Cela n'a pas été sans effet; si l'on n'a pas entendu la voix des coptes à cause de la délicatesse du sujet, la voix des femmes, elle, s'est fait entendre dans la presse. Les observateurs ont été pour leur part surpris par les candidatures du PND et considèrent qu'il y a là une «bombe à retardement " dont les effets ne manqueront pas de se faire sentir à un moment ou à un autre. (...) Un intellectuel copte qui a demandé à ne pas être cité pense que c'est une chute certaine du rôle des coptes dans l'histoire du mouvement national égyptien. Un des candidats coptes en question adopte la même position et justifie son attitude en disant qu'il est difficile, pour le PND, de présenter un candidat copte qui, de toutes façons, perdrait son siège ! L'autre candidat pense qu'il n'est pas besoin de discuter ce sujet, puisqu'un musulman représente un copte et vice-versa. (...)

Ce n'est pas l'avis d'Antoine Sidhom, du journal copte Watani, selon lequel c'est une logique inacceptable.

Si les uns pensent - dit-il - que le succès des candidats coptes est une aventure risquée, cela est démenti par le succès de ces candidats sur les listes du Wafd et des Libéraux constitutionnels avant 1952. Il y a également des circonscriptions à majorité chrétienne dans lesquelles coptes et musulmans cohabitent de manière fraternelle. Pourquoi ne pas y présenter des candidats coptes $7 \mathrm{Et}$ quel mal y a-t-il à ce que le PND présente une vingtaine de candidats coptes et que 10 ou 15 d'entre eux seulement soient élus? Parier des candidatures coptes comme d'une aventure risquée est une preuve d'impopularité du parti, car s'il était vraiment populaire, peu importerait que le candidat soit chrétien ou musulman.

Pour Mustafa Kamal Murad, chef des Libéraux, « les coptes auraient pu obtenir de nombreux sièges au parlement et les voix des électeurs ne constituent pas un problème. Mais les choix erronés du PND en ce domaine n'affectent en rien l'unité nationale.» Même avis chez l'écrivain islamiste Fahmi Huwaydi, pour qui « les candidatures du PNO n'ont pas été conçues comme il le fallait, mais ce qui affecterait l'unité nationale c'est que les coptes soient privés du droit de se présenter. Il ne convient pas, à cet égard, de 
se laisser entraîner dans un débat sur le nombre des sièges coptes, ce serait ouvrir la voie à la libanisation. » Pour Rifat Al-Sa'id, secrétaire du RP, «le PND a cédé au chantage des courants extrémistes sans prendre en compte les intérêts nationaux. La représentation proportionnelle est une fausse thèse imposée par le colonialisme (...) mais la proportion doit quand même être prise en considération.» L'historien Muhammad 'Imara présente une vision différente : «Ces dernières années, les coptes se sont retirés de l'activité publique, politique notamment. Les écrivains coptes se plaignent du fait que les électeurs musulmans ne votent pas en faveur des candidats chrétiens à cause de la montée du courant islamique. (...) Autrefois, Makram 'Ubayd se présentait à Sayyida Zaynab et était élu par les voix des musulmans. Aujourd'hui cela n'est plus possible, même si les coptes sont toujours les coptes et les électeurs les mêmes. En réalité, il était élu par les musulmans en tant que leader wafdiste, représentant d'un parti qui avait un projet national, et non en tant que copte. Une fois sorti du Wafd il n'a plus été réélu. (...) Aujourd'hui encore, un copte peut être élu s'il s'identifie au projet d'indépendance civilisationnelle adopté par la nation. Je n'en veux pour preuve que le succès, aux élections précédentes, d'un candidat copte en tête de liste de l'alliance islamique dans le fief des groupes islamistes militants. S'il ne s'était pas présenté sur cette liste, il n'aurait pas été élu. (...) Ce n'est pas une question de musulmans et de nazaréens, mais de projet de civilisation. L'électeur musulman pressent que l'isolement des coptes n'est pas seulement un renoncement à la chose publique mais aussi un refus de s'identifier à ce projet de civilisation. C'est là la vraie question qu'il convient de traiter. ${ }^{66}$

La réaction ne tardera pas à venir dans un « appel des coptes d'Égypte au président de la République ", publié cette fois dans le journal du RP et signé par un auteur extérieur au journal :

Les dirigeants politiques de l'État et du PND ont toujours présenté un certain nombre de candidats coptes. Nous avons été surpris par leur absence, notamment dans les circonscriptions où il $\mathrm{y}$ a une grande concentration de coptes, Chubra par exemple où ils sont plus de $55 \%$, tous membres du parti au pouvoir. Des deux candidats présentés, aucun n'est copte alors que ce siège est destiné traditionnellement à l'un d'eux. Pour assurer l'unité nationale entre chrétiens et musulmans et pour reconnaître le rôle des coptes, pour conforter la popularité du PND à Chubra, pour l'intérêt public et la sauvegarde de la démocratie, nous vous prions de reconsidérer les candidatures du PND. (...) Soyez certain que je respecterai vos décisions. ${ }^{67}$

«De quel droit l'auteur se permet-il de parler au nom des coptes? s'interroge un article publié dans le même journal :

Je pense que son enthousiasme et que sa naïveté risquent de faire croire au lecteur que les coptes recherchent hypocritement les bonnes grâces du parti au pouvoir, et je pense que nombreux sont les coptes qui se refusent à jouer ce jeu. (...) Peut-être l'auteur a-t-il voulu adresser un blâme personnel au parti qui ne l'a pas choisi comme candidat; il en a parfaitement le droit, mais nullement celui de présenter les coptes comme un groupe religieux qui fait allégeance à un parti donné. C'est une image dangereuse et abjecte. (...) Le fait qu'il y ait une grande proportion de coptes dans une circonscription signifie-t-il qu'il leur faut un candidat copte? C'est le début du glissement vers la représentation proportionnelle, refusée par les coptes depuis la Constitution de 1922. Pourquoi le PND n'a-t-il présenté que deux candidats coptes? C'est une question qui mérite d'être soulevée, surtout quand il s'agit d'un parti qui revendique une popularité fracassante dans un État ou les coptes représentent une part importante de la population. Le PND a-t-il tenté de traiter le phénomène de l'extrémisme par l'hypocrisie, en adoptant pour remède ce 
qui est la cause même du mal? Ou est-il incapable d'entendre la voix des masses qu'il prétend représenter? Si les coptes se sont fermés à la politique, un parti politique ne doit-il pas en rechercher les causes ?68 appartenances confessionnelles, toutes les opinions critiques à l'égard du PND sont unanimes pour exprimer refus et suspicion à l'égard de la nomination, par le président de la République, de représentants des catégories sous-représentées dans les élections. Bien qu'inscrit dans la Constitution, le droit du président de la République de nommer dix députés est perçu comme une fausse solution à de vrais problèmes et, de plus, est la manifestation même d'un népotisme préjudiciable à la fonction représentative et législative. A un point tel que le journal Al-Ahali rapporte les propos du patriarche de l'église copte Chenouda III qui, tout en invitant les coptes à dépasser la passivité politique, à s'inscrire sur les listes électorales et à voter pour le meilleur candidat, indépendamment de sa religion, assure qu'il n'est pas intervenu dans le choix des 
députés nommés par le président Moubarak ${ }^{71}$. C'est par cet acte - la nomination de dix députés - que prend fin généralement le dernier épisode des élections. Les députés nommés sont majoritairement - on s'en doute - des coptes : cinq dont une femme, et des femmes : trois dont une copte !( $\left.{ }^{72}\right)$

Les élections au quotidien

Tout cela ne suffit pas à rendre compte du déroulement d'une campagne électorale. $\mathrm{Si}$ l'on veut appréhender «le » politique dans son fonctionnement «sur le terrain », dans ses rapports avec «la » politique, dans ses détails, anecdotiques certes, triviaux peutêtre, mais sans doute bien plus révélateurs des modes de fonctionnement d'une société que le simple inventaire des positions des forces en présence, et si l'on reconnait, aux signes les plus individuels et les plus quotidiens, le statut de révélateurs d'une politique dont les limites restent à définir, il est possible, faute d'une lecture exhaustive, de repérer, sur le mode des "manières de faire ", des "manières d'élire » et de "se faire élire » qui, tout en témoignant de la distance qui sépare les masses tant de l'élite que de la contre-élite politique, n'en sont pas moins révélatrices de leurs points de rencontre, c'est-à-dire de la manière dont poids économique, appui familial ou communautaire, statut social, capacités et modes de mobilisation autour d'un thème, d'un individu, d'une identité, d'une revendication ou d'un mythe peuvent - dans une certaine mesure - alimenter l'élite politique et marquer de leurs empreintes la gestion des affaires de la cité.

Une campagne sans programmes

77 L'absence de programmes politiques - ils ne figurent que sur les journaux des partis et sont inexistants dans les rues - n'est pas un fart nouveau en soi. Mais elle s'est fait sentir encore davantage lors de ces élections. Ce n'est pas seulement le fait du scrutin uninominal ni la présence d'un grand nombre d'indépendants qui en sont la cause, mais il semble - à l'exception des candidats du RP qui, eux aussi, avaient compté, outre leur appartenance partisane, sur leurs assises locales - que nul n'ait ténu à faire de ces élections une bataille politique. Outre les slogans récurrents de la gauche (contre la loi d'urgence, pain et liberté, etc.), ses meetings politiques qui restent un fait exceptionnel dans cette campagne, ses banderoles dont on aurait pu croire, qu'elles dataient des élections précédentes, les quelques rares "l'islam c'est la solution", les programmes qui provoquent étonnement et sourire chez l'homme de la rue - quand ce n'est pas une franche hilarité - tels que la campagne "écologique» des Verts ou l'appel de Misr alFatat pour le creusement d'un nouveau lit du fleuve, on trouvera au mieux des allusions vagues (pour régler les problèmes de la société, pour la jeunesse...) et des promesses faites à la hâte aux électeurs pour régler tel ou tel problème spécifique au quartier, l'eau, l'électricité, les égouts ou les transports, et autant en emporte le vent de promesses électorales. La quasi-totalité des slogans prêchent les vertus personnelles des candidats, les plus célèbres étant les couples "générosité et piété », "droiture et sincérité », « don de soi et dévouement ».

Du nouveau dans la campagne

78 En revanche, les modes de campagne ne manquent pas de diversité : banquets organisés à la fin de meetings, où des professionnels dotés de dons particuliers et de voix puissantes ont damé haut et fort le nom du candidat fils de la circonscription qui, le temps de la campagne, se met en gallabiyya et dédaigne la veste, surtout s'il est candidat ouvrier ou paysan; photo et nom du candidat sur une carte qu'on ne pourra ni jeter ni déchirer car est imprimée au dos une sourate coranique ; inauguration de mosquées ; à 
Boulaq, cassette sur laquelle une chanson acclame le candidat du PND, ancien ministre de la Culture ; à Minuf, autre cassette d'un célèbre chanteur populaire pour un candidat millionnaire au chéquier généreux; bandes vidéo pour immortaliser tel meeting; tel candidat, boulanger de son métier, distribuant le pain, tel autre offrant des savons avec sa photo car il « lave plus blanc", la liste est longue... Elle le serait plus encore si on ajoutait la liste des symboles qui permettent aux illettrés d'identifier le candidat qu'ils choisissent, pratique qui permet d'avoir un avantage sur le concurrent ou, au contraire, de se voir couvrir de ridicule car le croissant de lune est infiniment plus noble que la cruche. Au dire de Rose al-Yusuf, tel candidat a recourt aux méthodes des groupes islamistes de l'université. Il organise des concours religieux dont les prix ne sont ni des corans ni des livres pieux comme on s'y attendrait, mais des réfrigérateurs, des machines à laver et autres appareils électroménagers qui poussent les habitants du quartier à se bousculer pour y participer. ${ }^{73} \mathrm{La}$ campagne électorale mobilise de tels effectifs et de telles sommes - l'enjeu est sans doute à la mesure du prix qu'on y met qu'un professeur de sciences politiques futé, visiblement impressionné par les campagnes à l'américaine, publie l'annonce suivante: "Comment gagner un siège à l'Assemblée du Peuple? Aux cadres populaires désirant une préparation rapide et efficace aux élections, nous présentons notre expertise pratique et scientifique pour planifier et gérer la campagne électorale. ${ }^{74}$ Justification de ce projet sans précédent à l'échelle du monde arabe: «La campagne électorale nécessite une stratégie. Aujourd'hui, la démocratie est une industrie lourde; il y a des professionnels qui la pratiquent de manière improvisée. Nous l'organisons de manière scientifique. $»^{75}$

Qui paye?

79 Qui dit « industrie lourde » dit capitaux. Comment apprécier au juste l'impact de cet élément qui semble peser lourd sur le déroulement de la campagne électorale, si ce n'est d'après les résultats?

Il faut savoir au départ qu'un « élu de la nation » est rémunéré de la manière suivante : avec une session parlementaire de 7 mois par an comportant en moyenne 42 séances payées chacune $50 \mathrm{LE}$, auxquelles s'ajoutent environ 30 sessions des divers comités de l'Assemblée, payées entre 20 et $30 \mathrm{LE}$, et une indemnité mensuelle de $100 \mathrm{LE}$, un député aura "gagné » en moyenne 4000 LE par année de mandat, chiffre exorbitant par rapport à la moyenne des salaires mais ô combien dérisoire comparé aux frais de la campagne électorale, « payés le plus souvent par les supporters du candidat si l'en en croit un ancien député $» .^{76}$ II faut croire qu'être membre du parlement donne droit à d'autres privilèges et "facilités»: le journal Al-Ahali note qu' "un nombre sans précédent de millionnaires se sont présentés à ces élections, auxquels s'ajoutent nombre de candidats impliqués dans des procès en cours devant la justice. Sur 20 d'entre eux, en instance de jugement devant la cour d'Assises, 5 ont été élus et ont droit à l'immunité parlementaire. $\gg^{77}$ Rose al-Yusuf titre : « Le plus grand trafiquant de drogue se présente aux élections et pourrait être élu. $\aleph^{78}$. Al-Ahali trace le profil d'un de ces candidats :

81 «Les affiches du candidat, ancien député et membre du PND, annoncent: «0 Dieu, viens en aide à ton pauvre serviteur." Question pauvreté, il s'est enrichi par des moyens illégaux, possède plusieurs milliers de feddan extorqués à l'État, plusieurs résidences et se présente en candidat ouvrier! Profitant du fait qu'il était membre du conseil d'administration d'une coopérative de construction de logements, il a distribué des appartements aux responsables plutôt qu'aux membres de la coopérative, a 
fabriqué de faux documents, s'est rendu coupable de fraude fiscale et a construit 60 immeubles sans permis de construction. (...) C'est seulement la semaine dernière que des mesures ont été prises contre lui, car il a insisté pour se présenter comme concurrent du candidat du PND en prétextant qu'il avait besoin de l'immunité parlementaire davantage que lui. (...) La levée de l'immunité est lente à obtenir et exige plusieurs démarches complexes dont l'issue dépend du gouvernement. Toute plainte contre un député est transmise au ministre de la Justice qui, à son tour, la transmet à la commission législative de l'Assemblée à majorité gouvernementale, laquelle peut décider de ne pas donner suite ou de soumettre la plainte à l'Assemblée. A son tour l'Assemblée - à majorité gouvernementale également - peut rejeter ou accepter la demande. Le plus souvent, celle-ci se perd dans le dédale des procédures. $\gg^{79}$

Scrutin uninominal oblige, le PND avait annoncé que ses candidats assumeraient les frais de leur campagne et que le parti n'avait pas prévu de budget à cet effet. ${ }^{80}$ Le ministre de l'Intérieur avait annoncé pour sa part les décisions relatives au déroulement de la bataille électorale: affichage limité à des endroits précis dans chaque circonscription; campagne ne devant pas comporter des thèmes contraires à l'unité nationale, la paix sociale et la souveraineté de la loi: pour les réunions publiques organisées sous des chapiteaux, demande à effectuer auprès des autorités 24 heures au moins à l'avance, décision en fonction des impératifs de sécurité. A cela, le correspondant d'Al-Ahram ajoute que pour garantir le bon déroulement de la campagne, des forces de police sont mises à la disposition des candidats qui le souhaitent dans les quartiers ou il y aurait des risques d'affrontement. ${ }^{81}$

83 La disposition la plus éloignée des pratiques en vigueur semble être celle qui limite les frais de la campagne électorale à 5000 LE pour garantir l'égalité entre candidats. Ils sont estimés à $30000 \mathrm{LE}$ par Al-Akhbar (accueil, repas, location de voitures pour le groupe du candidat, imprimés et matériel de publicité) ${ }^{82}$. Al-Wafd estime ces frais à plus de $70000 \mathrm{LE}{ }^{83}$. Pour le seul quartier de Boulaq, on dénombre mille arcades (échafaudage portant le nom du candidat) à raison de 500 LE chacune, soit un total d'un demi million ${ }^{84}$. Voici la description qu'en donne Al-Ahram : «Les rues ont disparu sous une forêt d'affiches et de banderoles. Les géants fortunés envahissent Boulaq: propriétaires de sociétés d'export-import, commerçants en fer, en tôle, en viandes, en chaussures, bouchers, tous ceux-là se sont présentés contre les candidats du PND pour occuper l'espace vacant. Si l'assaut a recouvert les traits de Boulaq, il a donné naissance à un slogan, "Boulaq aux Boulaquiens", slogan que reprennent les jeunes en annonçant leur refus que des candidats argentés distribuent à la sortie des mosquées leurs tracts publicitaires, agrémentés chacun d'un billet d'une livre... $»^{85}$

La fraude et la violence

84 La présence de ces riches candidats ne se limite pas seulement aux fastes déployés durant la campagne électorale. On évoque l'achat des voix à l'entrée des bureaux de vote, le prix augmentant à mesure qu'approche l'heure de la fermeture ${ }^{(86)}$, et on parie des vingt livres versées en acompte pour une voix promise ${ }^{87}$. Un candidat de Minufiyya aurait annoncé qu'il consacrait 4 millions de livres à sa campagne ${ }^{88}$. Par leur influence, certains d'entre eux seraient allés jusqu'à faire basculer le contenu des urnes. Cas célèbre, celui de Hamdi Al-Sayyid, candidat du PND dans la circonscription d'Héliopolis, quartier bourgeois avec une proportion élevée d'universitaires, où le candidat du parti, personnalité célèbre, ex-président du syndicat des médecins, connu par ses activités sociales dans les clubs huppés du quartier, cumulant respectabilité sociale et crédit 
politique, se retrouve battu alors que le dépouillement lui était favorable, par un richissime homme d'affaires totalement inconnu, vivant en Égypte depuis dix ans et ayant acquis sa nationalité il y a deux ans. Le candidat rejeté accuse le juge responsable du déroulement du scrutin d'avoir falsifié les résultats pour permettre au concurrent de « piquer » sa circonscription ${ }^{89}$.

C'est que la fraude n'est pas qu'une pratique gouvernementale. C'est une des leçons les plus importantes de ces élections. Le fait n'est pas nouveau en soi, mais il a été mis en exergue du fait de la "propreté » réelle ou supposée des élections, en tout cas promise par le gouvernement "Propreté » reconnue par la gauche, qui a réussi à placer quelques candidats et ne déplore que "des cas isolés d'interventions dans le scrutin, dus aux potentats locaux", alors que pour les partisans du boycott il s'agit des pires élections de l'histoire égyptienne.

En tout premier lieu vient la technique fort célèbre du «bouclage", une spécialité égyptienne, un classique des élections pratiqué allègrement sous tous les pouvoirs, non seulement sur ordre des autorités mais aussi à l'initiative des responsables de bureaux de vote désireux de marquer leurs sympathies pour tel ou tel candidat. Cela consiste à cocher les bulletins de vote des personnes décédées, des émigrés et des absentéistes, procédé d'autant plus praticable que les listes des électeurs ne sont pas mises à jour. Un « spécialiste » s'en explique :

Les meilleurs moments pour le faire sont d'abord le matin, après la première heure d'ouverture des bureaux de vote, avec la complicité de la police et la présence d'un grand nombre de partisans du candidat. On coche les bulletins seulement, sans cocher les noms sur les listes électorales et en gardant une réserve de $15 \%$ des bulletins jusqu'à la dernière heure avant l'ouverture (au cas où des électeurs arriveraient. NDLA) Faute de pouvoir le faire le matin, cela se passe en fin de journée, après entente avec le responsable du bureau de vote. (...) Mais il faut veiller aussi à ce que les concurrents ne fassent pas la même chose dans les autres bureaux. En somme, boucler ici et déboucler là bas..$^{90}$

Parmi les conflits dus aux irrégularités de déroulement du scrutin, il en est un sur lequel la presse s'est longuement attardée et qui ne manquait pas de saveur. Il opposait en effet un candidat indépendant, magistrat et frère aîné du premier ministre, pas moins, à un potentat du PND, 'Attiyya Al-Fayumi, alias «la baleine", lié à plusieurs affaires louches - vente de terrains appartenant à l'État et dilapidation des deniers publics - auxquelles il doit son surnom. Constatant des irrégularités dans le déroulement du dépouillement, refusant que celui-ci ait lieu dans un local à proximité du poste de police et s'opposant ainsi à son confrère, le juge chargé du contrôle des opérations, le magistrat obtient la reprise du dépouillement, auquel il participera personnellement : l'opération durera cinq jours sans interruption, retardant l'annonce des résultats au niveau national. Le candidat plaignant l'emporte finalement sur son concurrent avec une différence de 2000 voix. Le ministre de l'Intérieur déclarera par la suite que son ministère n'est pour rien dans cette affaire et que les résultats n'ont pas été affectés par le fait que le conseiller 'Adil Sidqi soit le frère du premier ministre: c'est un juriste qui connaît ses droits et a tenu à les faire respecter ${ }^{91}$.

Dans le débat sur la régularité des élections, deux thèmes dominent : la neutralité de la police et l'intervention des appareils administratifs. En dépit de quelques réserves concernant des incidents localisés, le RP reconnaît que la police a adopté une position neutre alors que pour la presse des partisans du boycott, cette prétendue neutralité n'était qu'une feinte destinée à mieux couvrir la fraude. Tout en relatant les épisodes 
d'interventions en complicité avec .la police contre les candidats du RP, à Alexandrie, à Mit-Ghamr, et en notant la recrudescence des incidents violents dus soit à la police, soit aux " unités spéciales ", commandos privés constitués par certains candidats, Al-Ahalî publie les déclarations du chef du RP selon lesquelles « le bénéfice réel de ces élections est que pour la première fois, la police a adopté une position neutre : elle n'a pas tenté d'exercer des pressions sur les 'umda de villages, comme c'est le cas d'habitude et à quelques exceptions près. Cela dit, la police est toujours impuissante face au phénomène du bouclage. (...) Les interventions de certains gouverneurs ont été flagrantes, mais ce n'est pas le cas pour tous. Comparées aux élections précédentes, c'est un pas en avant. ${ }^{92}$ Même verdict selon Philippe Gallab, journaliste fameux de AlAhalî :

Le succès de candidats du RP, du courant nassérien et d'opposants indépendants et l'échec de certaines figures gouvernementales est une surprise réelle. Il est vrai qu'il y a eu des cas d'interventions flagrantes contre les candidats du RP, les indépendants, voire les candidats du PND eux-mêmes et dans lesquelles étaient impliqués tant certains responsables de la police que des chefs de bureaux, de commissions et des gangs criminels spécialisés. Mais il ne s'agissait pas tant, le plus souvent, de la mise en œuvre de directives officielles que des manifestations d'une corruption qui s'étend à plusieurs niveaux, et de l'absence des garanties revendiquées par l'opposition. ${ }^{93}$

II en est autrement pour le journal Al-Wafd qui, relatant les cas de centaines de bulletins de vote favorables à un candidat, découverts dans la poubelle d'un poste de police, ou présentant des listes électorales sur lesquelles on a coché les voix des morts, ressuscites pour l'occasion afin de manifester leur soutien au PND, donne sa version sur les incidents de Faraskur et remet en cause le rôle de la police, tout en soulignant un degré de violence sans précédent : « Etrange neutralité que celle des forces de police. Elle leur interdit de pratiquer la fraude mais les autorise à laisser faire les bandes de fier-à-bras et de fraudeurs. (...) A Roda, un village de Faraskur, les habitants se sont révoltés contre le truquage en faveur du candidat PND ; ils ont mis le feu à un poste de police, ont brûlé trois cars de police, un car blindé et deux voitures privées. Les forces de sécurité ont répondu par des coups de feu qui ont fait cinq morts et cent trente blessés, dont vingt dans un état grave. $»^{94} \mathrm{Al}$-Wafd rapporte également la mort de 8 personnes dans des échanges de coups de feu entre les partisans de deux candidats concurrents, à Qena, au deuxième tour ${ }^{95}$.

\section{Messieurs les ministres en campagne}

91 Outre ces « incidents », la presse d'opposition relate l'intervention de l'administration tant dans le déroulement de la campagne que dans les opérations de vote, intervention qui s'est manifestée notamment par la mobilisation de l'infrastructure de la fonction publique en faveur des ministres candidats (ce type de candidatures est autorisé par la Constitution, qui opte pour une séparation souple entre pouvoir législatif et pouvoir exécutif). Al-Musawwar décrit ainsi la tournée d'un ministre :

Sa circonscription n'est pas comme les autres, elle a de la chance, et les habitants sont certains que le ministre pourra réaliser toutes ses promesses. (...) A l'entrée du plus gros village s'agglutine une foule d'hommes, de femmes et d'enfants. Le cortège est précédé par une troupe de musiciens qui joue des airs populaires; pancartes de soutien sur les tracteurs, youyous des femmes, haut-parleurs qui évoquent les réalisations du ministre. (...) La visite commence par la maison $d u$ 'umda (...) Des milliers de demandes à signer ; pour ces électeurs, le ministre n'est pas seulement spécialisé dans les questions qui relèvent de son ministère : il peut résoudre tous les problèmes; l'électricité, l'eau d'irrigation, les exonérations 
d'amendes, les avoirs de ceux qui rentrent d'Irak, la mosquée du village, la cabine téléphonique... ${ }^{96}$ malgré l'attribution d'un temps de parole égal aux représentants des partis, on ne peut faire abstraction de la manière avec laquelle la presse officieuse souligne l'échec de certaines grandes figures du PND, notamment neuf des secrétaires régionaux du parti. Il faut croire que ces échecs sont interprétés comme un triomphe de la démocratie, une preuve supplémentaire de la régularité des élections. Au-delà, c'est peut-être un appel au PND à se renouveler et à épurer ses rangs. En toile de fond, les conflits qui ont opposé les candidats du part aux membres non choisis qui se sont présentés en indépendants. PND contre PND, c'est là que la bataille a été la plus acharnée, révélant les conflits de pouvoir et les divergences, notamment celtes qui ont opposé les gouvernerais à la capitale ou les gouverneurs aux choix du secrétaire général du PND. A Mahallia, un tract du candidat PND accuse les autorités locales et les dirigeants du parti de soutenir son concurrent indépendant $\left({ }^{100}\right)$; le secrétaire générai du parti rejette les propositions de candidature du gouverneur de Minya, et parti, conseils municipal et exécutif se divisent et soutiennent des candidats différents ${ }^{101}$. C'est sans doute dans le gouvernorat d'Isma'iliyya que le conflit est le plus grave, puisqu'il oppose un candidat officiel du PND, une des grandes figures du courant nassérien récemment entré au parti, à un puissant gouverneur qui s'oppose à cette candidature et soutient ouvertement, par voie de tracts, manifestations, actes de vandalisme contre les banderoles du candidat officiel et intimidation de ses partisans, une autre candidate ${ }^{102}$. Qu'on se rassure : la candidate en question a nommée par la suite par le président de la République. 

l'état de sa discipline, c'est le choix de certains candidats du parti au pouvoir que la presse d'opposition met en exergue. Ainsi le journal Al-Wafd écrit-il : «A ses attributs, le PND pourra ajouter qu'il est le parti des antécédents criminels. Il a présenté comme candidats des rois de la pègre, du vol et du pillage dans les gouvernorats, en dépit des jugements prononcés par la justice : il a présenté à nouveau huit de ces anciens élus qui avaient été privés de l'immunité parlementaire et trente candidats inculpés dans divers procès. $»^{103} \mathrm{Al}$-Wafd rapporte également certains propos du ministre de l'Intérieur qui aurait reconnu que deux des députés élus avaient été inculpés pour trafic de drogue. Il a assuré, selon le même journal, qu'il s'était opposé à la candidature de quatre trafiquants mais que le parti y tenait en raison de leur « popularité » ${ }^{104}$.

Profil d'un député

ers éments relatifs au déroulement de la campagne et aux positions des forces en présence permettent d'identifier un contexte et de décrire une conjoncture. Cependant ils ne suffisent pas à rendre compte de ce qui guide le choix de l'électeur, élément central et enjeu de cette bataille électorale dont il semble pourtant si éloigné. Si les observateurs des élections égyptiennes s'accordent sur l'impact limité des programmes et de la coloration politique des candidats, ils identifient quelques éléments qui, sans être explicatifs, permettent d'y voir plus clair. Le journal AlGumhuriyya évoque le Rapport du Centre d'études stratégiques d'Al-Ahram qui, analysant les élections de 1987 qui se sont déroulées suivant un panachage de scrutin de listes de partis et de candidatures indépendantes, met l'accent sur le rôle de la propagande électorale. Pour les élections de 1979, la même publication signale l'impact infime de la campagne électorale, comparée à d'autres facteurs. En tête de ceux-ci, les services que le candidat est en mesure de rendre à sa circonscription, les 'açabiyya familiales ou communautaires, (acteurs qui jouent indépendamment de la propagande électorale du concurrent et des appartenances partisanes : ces éléments viennent en dernier. Pour un candidat du PND,

La bataille électorale devrait s'engager autour des idées et des politiques du parti auquel appartient le candidat, de manière à ce que les élections soient le reflet de la majorité souhaitée par le peuple. Et cela quels que soient la tête du candidat Ou ses rapports personnels avec les électeurs. Dans les faits, cela se passe autrement; nombreux sont ceux qui donnent leur voix au candidat qui pourra leur rendre des services purement personnels, un poste, une mutation ou des services locaux comme la réparation d'une route, l'installation d'un réseau d'électricité dans le village. Il y a une confusion entre les élus locaux des conseils municipaux et ceux de l'Assemblée. (...) Résultat : élection au parlement d'individus qui ne sont aptes qu'à un travail au niveau local. (...) Il faut ajouter à cela que l'élection est quelquefois due au simple fait qu'un tel appartient à telle famille ou est né dans tel village. Plutôt que de choisir le meilleur, on choisit le plus proche. Restent enfin les nombreux électeurs qui se laissent impressionner par la propagande électorale de candidats qui vont jusqu'à publier des livrets portant exclusivement sur leur personne et leur biographie, sans la moindre mention de son programme politique et des idées de son parti.

Pour les élections de 1990, le candidat nassérien indépendant Dia' al-Din Dawud pense que de nouveaux facteurs entrent en jeu : «le rôle positif d'une jeunesse accablée par le chômage et l'absence d'espaces collectifs, la recherche de personnalités nouvelles capables de prendre à cœur les problèmes quotidiens et le sentiment que jusqu'ici, l'opposition a été incapable de transformer les slogans de sa campagne en propositions

Égypte/Monde arabe, 4 | 1990 
alternatives concrètes. » A ces divers facteurs, Khalid Muhy-l-Din, chef du RP, ajoute le poids du dispositif des conseils municipaux :

«Leurs prérogatives sont nombreuses et de la plus haute importance. Nous souhaitons que leur rôle soit favorable à la démocratie. ${ }^{105} \mathrm{~A}$ noter également les propos $\mathrm{d}$ ' AlSiyasi, qui écrit dans un tout autre but: «La majorité de la population égyptienne vit dans des villages, dans des villes à caractère rural ou dans des cités ouvrières. Majorité ignorante et inconsciente $\mathrm{du}$ « $\mathrm{b}-\mathrm{a} \mathrm{ba}$ » de la politique et de ce que c'est que de donner sa voix à ceux qui la méritent. Leur seul souci est le gagne-pain ; le jour des élections, ils se rendront au bureau de vote conduits par les 'umda pour voter pour le candidat qu'on leur aura désigné. Généralement, ils y vont sous la menace ou parce que le candidat est un proche, quelle que soit sa tendance politique, ou encore parce que les élections sont pour eux un spectacle amusant. Une minorité d'entre eux saisit la portée des élections, non comme opération démocratique mais parce que le succès de leur candidat leur garantit intérêt et influence. Seule une minorité parmi la minorité appelle à la démocratie, désireuse de faire fonctionner la société selon ses théories. Le vacarme qu'on entend avant les élections provient d'une poignée d'intellectuels aux voix puissantes, qui rêvent de la démocratie occidentale sans se demander si les conditions de sa mise en œuvre existent. L'ouvrier ou le paysan égyptien, qu'ont-ils de commun avec un ouvrier ou un paysan français, suédois ou américain? ont-t-ils le sens de la citoyenneté comme responsabilité, et un contact avec la technologie moderne qui les habitue à faire fonctionner leur cerveau de manière logique et rationnelle ? (sic) »106

Curieuse image que donne un journal proche du parti au pouvoir de la majorité que représente le PND. La question reste posée : faut-il prendre en compte de nouvelles variables qui influenceraient le vote, ou est-ce toujours en fonction des facteurs de 'longue durée', des « pesanteurs » d'une société qui persisterait à voir dans la politique une sphère extérieur à elle, que les jeux se font? (Selon Al-Wafd, des statistiques récentes montreraient que les membres des partis en Égypte représentent 5,5\% de la population. $)^{107}$

101 Faute de pouvoir répondre à cette interrogation, les exemples ne manquent pas qui témoignent de la vitalité et du renouvellement de mécanismes de cooptation présumés traditionnels, jeu des solidarités communautaires, échange de services ou leadership exceptionnel de certains «candidats historiques ». C'est le cas du vétéran EIwi Hafez, membre de toutes les organisations politiques depuis 1952, la dernière en date étant le Wafd dont il vient de se séparer à la suite des élections. Il a représenté sa circonscription, Al-Darb Al-Ahmar, depuis 1960, avec une seule interruption sous l'exministre de l'Intérieur Zaki Badr: "Le secret de mes rapports avec les gens est qu'en 1954, j'ai monté un camp d'entraînement militaire dans le cadre de la résistance contre l'occupation, qui a regroupé 50000 des habitants du quartier. Aujourd'hui, ils sont 50 000 pères et mères auxquels s'ajoutent leurs enfants. Dans mes rapports avec la population du quartier, ils ne me posent jamais de questions sur mon appartenance politique ou idéologique. $»^{108}$ Autre exemple non célèbre mais combien éloquent, celui de Zayn Al-Sammak, ingénieur/prédicateur, membre du RP et candidat à Alexandrie. Dans ce cas, l'immersion locale du candidat, si profonde soit-elle, a du mal à tenir face à la ténacité de certaines solidarités communautaires. C'est le cas dans cette circonscription de Ghorbal, quartier pauvre, réputé marginal, à forte densité, où se concentre une population venue de Haute Égypte pour travailler dans le bâtiment : « Ils sont structurés - écrit le candidat - selon des 'açabiyya et encadrés par des associations 
qui contrôlent leurs membres, ont des cartes d'électeurs, et on peut difficilement se faire élire sans le crédit des associations et des 'açabiyya. C'est à la fois facile et difficile. J'ai quand même décidé de me présenter parce que c'est là que je suis né et que j'ai vécu, jusqu'à l'âge de 28 ans. (...) Voilà plus de trente ans que je me retrouve tous les soirs dans le centre islamique de Ghorbal (...), d'autant plus heureux que ces élections me permettent de poursuivre ma tâche, qui est de propager conscience politique et conscience religieuse en même temps. Les habitants de ce quartier peuplé de mosquées et d'églises aiment et respectent l'homme de religion. (...) lis ont cette courtoisie discrète des gens du Sa'id et quand ils aiment, c'est avec sincérité. (...) Toutes les fois que je rencontre l'un d'eux, il me dit qu'il a écouté le cours religieux que je donne à la mosquée, que son fils est à la crèche du centre ou qu'il s'est rendu à la salle de cérémonie du centre pour une occasion ou une autre. (...) Ghorbal cherchera-t-elle à se faire une place dans le monde de la démocratie? $»^{109}$ Témoignage saisissant par sa lucidité d'un candidat qui, malgré sa présence dans la vie d'une communauté locale, transplanté loin de son lieu d'origine, reconnaît qu'il n'en fait pas partie, qu'un abîme le sépare de ceux au nom desquels il voudrait parier et par rapport auxquels il reste à l'extérieur. Il espère pourtant, et ce que raconte Sammak en dit plus long que beaucoup de discours démagogiques et d'analyses scientifiques.

Dans d'autres cas, la question est plus simple à régler. Le siège à l'Assemblée est un héritage, un bien familial qu'on se transmet de génération en génération. Al-Ahram donne une liste d'une vingtaine de candidats dont les pères, voire les grands-pères, ont été députés. «Je pensais, écrit le journaliste, que c'était un phénomène négatif : mais en l'absence d'un milieu propice à la formation de cadres politiques, la famille devient le seul terroir de formation des hommes politiques qui vont immortaliser le nom de famille, entre autres choses. (...) » Dans ce même article, Milad Hanna, ancien député de gauche, dresse le portrait d'un député : "C'était un solitaire en tarbouche et habit de notable rural. Je n'ai jamais su son nom, jamais je ne l'ai entendu parier. Il faisait partie de ces ruraux aisés qui sont dénués de toute culture, mais règlent des problèmes. Ceuxlà, ils sont prêts à s'orienter vers n'importe quel parti, selon la direction du vent. (...) Une sorte de contrat social les relie à tout système quel qu'il soit : "L'État nous donne l'influence et nous lui donnons les liens avec le village »; la pensée politique, ils ne connaissent pas. $»^{110} \mathrm{Ce}$ que décrit Milad Hanna est un portrait robot, le modèle même $\mathrm{du}$ député. Ce modèle en évoque un autre, celui tracé "dans un moment d'enthousiasme" par Léonard Binder ${ }^{111}$. Le "second stratum" tiendra-t-il encore longtemps? Aurait-il réussi une nouvelle reconversion?

Le paradoxe du votant

S'il n'est pas aisé de tracer le ou les profils d'élus, tracer celui de l'électeur est encore plus difficile, surtout quand l'abstention domine largement et que le vote, qui est pourtant la règle du jeu, devient exception. Cependant, seul un élément permet de pondérer tout ce qui vient d'être dit, c'est la participation des électeurs. Débat récurrent que l'on retrouve à chaque élection, avec les deux volets de rigueur: polémique sur le taux de participation annoncé par le gouvernement et discours alarmiste/volontariste à prétention sociologique sur l'abstentionnisme, source de tous les maux. Cette fois-ci, il fallait compter également avec les partisans du boycott, qui ont interprété l'abstentionnisme comme une réponse à leur appel et une confirmation de plus de sa pertinence. 
104 Il faut noter au départ que la moyenne nationale de participation annoncée par le ministre de l'Intérieur était de $44,95 \%$. Dans son communiqué, le ministre indiquait également une constante électorale, la supériorité du taux de participation dans les villages proportionnellement aux villes. Il atteint $85 \%$ dans certaines régions de Haute Égypte, $75 \%$ dans le nord contre $7 \%$ seulement dans certaines circonscriptions du Caire $^{112}$.

105 Le journal Al-Wafd parle de contradictions dans les déclarations gouvernementales au sujet du taux de participation: "Si nous admettons que 7 millions d'électeurs ont voté, ce chiffre ne peut rendre compte à lui seul du taux de participation réelle. Le corps électoral devrait correspondre au chiffre des citoyens adultes de 18 ans et plus. Or les chiffres confirment un écart énorme entre les deux. Le nombre des inscrits, 16 millions, représente au plus $43,75 \%$ du corps électoral, qui lui est de 37196836 citoyens. Cela veut dire, en d'autres termes, que la proportion des votants dans le corps électoral est de $18,8 \%$, et qu'ils représentent $12,28 \%$ d'une population de 57 millions. ${ }^{113}$

Le même journal évalue le taux de participation au premier tour à $10 \%$ et au deuxième tour à $5 \%$ des électeurs ${ }^{114}$ et publie, quelques jours plus tard, un « communiqué du Wafd à la nation : Merci aux électeurs qui ont boycotté les élections. (...) Le Wafd est fier de ce peuple combattant et endurant qui a répondu à l'appel au boycott. La majorité a refusé de participer à ces élections, les statistiques officielles confirment que 5 à $12 \%$ seulement des électeurs dans les villes ont participé aux élections. Certains candidats ont été élus à $3 \%$ des voix des inscrits, et nul ne pourra prétendre que cette Assemblée représente le peuple. $»^{115}$

$107 \mathrm{Au}$ delà du fait que les pratiques de bouclage et que les listes électorales non remises à jour interdisent d'estimer de manière satisfaisante le taux de participation, et indépendamment de l'interprétation de l'abstention comme réponse à l'appel au boycott, reste le fait que le vote est une pratique exceptionnelle, même si l'abstention des inscrits donne lieu théoriquement à une amende d'une livre 1 Entre l'abstentionnisme et le rejet du politique, la distance est grande, puisque ce rejet est lui-même politique. En 1984, Port-Saïd a donné 51\% de ses voix à l'opposition. En 1987, sur les six opposants que comptaient l'Assemblée, trois étaient des députés de PortSaïd ${ }^{116}$. Etrange ville que Port-Saïd, liée dans les mémoires à la fois à son histoire de ville résistante et de zone d'infitah (ouverture économique) précoce, frappée aujourd'hui par la récession. Le journal Al-Ahram décrit ainsi un aspect de la campagne électorale qui s'y déroule :

Des groupes de la jeunesse dorée paient la caution de candidature (200 LE, NDLA) à certains candidats qu'ils choisissent. Ils organisent ensuite des réunions électorales qui durent jusqu'à l'aube au milieu des rires, des slogans humoristiques. Ils font de l'occasion un prétexte à s'amuser et sont allés jusqu'à imprimer des tracts et des programmes électoraux aberrants. (...) Un exemple honteux que l'on voit à PortSaïd pour la première fois et qui pose la question des raisons de cette conduite pathologique qui dépasse le simple refus passif pour aller jusqu'au refus sarcastique. Cela est d'autant plus regrettable que ces réunions, qui se déroulent au siège électoral du candidat, sont organisées et gérées par une jeunesse instruite et aisée. ${ }^{117}$

108 II n'est pas sans intérêt de noter qu'au nombre des "spécialistes » consultés par la presse, donc autorisés à porter un jugement sur la question, viennent en premier les psychologues. Muhammad Cha'Ian, le plus médiatique d'entre eux, déclare: «Le citoyen pressent que sa voix n'a aucune valeur, que son rôle dans le jeu est défini 
d'avance. Nous avons appliqué les formes de la démocratie sans en retenir le fond. Cette notion doit acquérir sa crédibilité en commençant par le bas. ${ }^{118}$ Un autre psychologue évoque la crise de confiance et l'absence d'identification à un parti qui a phagocyté plus d'une fois la volonté populaire, raisons pour lesquelles le citoyen égyptien est atteint d'un "négativisme dépendant ", absence de réaction à tout ce qui touche aux questions politiques et nationales, si bien qu'il ressent une satisfaction psychologique à tourner le dos aux élections, pour ne pas endosser la responsabilité d'avoir participé à l'ascension de ces députés qu'il n'a pas choisis. Un troisième enfin ajoute que les « conduites politiques des Égyptiens sont frustrées » (!) puisqu'ils savent que les choses suivront leur cours, qu'ils interviennent ou non. Réalité insoutenable à cause de laquelle le citoyen n'a plus de respect pour sa propre voix. Ajoutons à cela que les élus qui soutiennent inconditionnellement le gouvernement dorment pendant les séances, s'absentent ou recherchent leurs intérêts privés ne lui inspirent pas confiance $^{119}$. Le sociologue Abd al-Hadi Al-Guhari explique l'abstentionnisme en ces termes: "Nous avons adopté une vote qui, d'une façon ou d'une autre, a renforcé l'individualisme et l'égoïsme, et cela a poussé beaucoup de gens à se détourner de la participation. Nous avons pris les décisions en l'absence des intéressés sous prétexte, que nous étions mieux placés pour définir leurs intérêts ; au point que chaque individu, pressentant que sa destinée est entre les mains d'autrui, a préféré chercher ses intérêts particuliers tout en affichant son allégeance au groupe. $»^{120}$

Frustrés les Égyptiens? Individualistes? Des raisons de s'abstenir, de se détourner du spectacle, il y en aura toujours, et de bonnes. Faute de les connaître toutes, ne devraiton pas rechercher plutôt les raisons obscures de cette pratique étrange et marginale qu'est le vote ? Peut-être comprendrait-on mieux, alors, le paradoxe du votant.

\section{NOTES}

1. Pizzorno A., "Sur la rationalité du choix démocratique », in Pierre Birnbaum \& Jean Leca, Sur l'individualisme, FNSP, Paris, 1986, p. 346.

2.Al-Ahram 2/1/90.

3.Al-Ahram 8/12/90. Ces résultats ne comprennent pas ceux de 4 circonscriptions pour lesquelles les élections ont été reportées. C'est ce qui explique l'écart entre le pourcentage et le nombre de sièges.

4.Al-Musawwar 19/11/90, Faruq Abaza, p. 44-45.

5.Al-Ahram 13/11/90. Les chiffres attribués par le ministère de l'Intérieur aux élus des partis du boycott ne sont pas reconnus par ces partis.

6.Al-Ahram 12/11/90, Salah Muntasir.

7.Akhir Sa'a 10/10/90, Samir al-Husayni, p. 8-9.

8.Al-Wafd 11/10/90, Mahmud al-Charbini.

9.Al-Wafd 17/10/90.

10.Al-Wafd op. cit.

11.Al-Wafd op. cit.

12.Al-Wafd op. cit. 
13. Mayo 12/11/90.

14.Al-Ahram 12/10/90. Salah Muntasir.

15.Voir à ce sujet Alain Roussillon : «Les Nouveaux partis politiques ", Égypte/Monde arabe, 2/ 90, p. 123-142.

16. Al-Wafd 29/10/90.

17.Al-Wafd 29/10/90.

18.Al-Cha'b 23/10/90.

19.Al-Wafd 26/11/90, Nabil Al-Hilali.

20.Al-Cha'b 23/10/90, 'Adil Husayn.

21.Al-Wafd 1/11/90, Imad al-Ghazali.

22.Al-Ahrar 26/11/90.

23. Rose al-Yusuf 29/10/90, Ibrahim Khalil, p. 10-13.

24.Al-Gumhuriyya 29/11/90.

25.Rose Al-Yusuf 29/10/90, Abd al-Qadir Chuhayto p. 13.

26.Al-Ahram al-lqtiçadi 12/11/90, Bahyil-Din Chu'ayb, p. 44-46.

27.Al-Ahram 27/10/90, Salama Ahmad Salama.

28. Mayo 24/10/90, Muhammad Isma'il 'Ali.

29.October 4/11/90, 'Abd al-'Azim Ramadan, p. 26-28.

30.Al-Akhbar 27/10/90, Samir Abd al-Oadir.

31.Al-Ahram 28/11/90, Salah al-Din Hafiz.

32.Al-Cha'b 30/10/90.

33.Al-Ahali 26/12/90, Sàîd Isma'il 'Ali.

34.Al-Ahali 31/10/90, Muhammad Sayyid Ahmad.

35.Al-Ahram 11/11/90, Abd al-'Azim Darwich.

36.Al-Ahram 7/12/90, Sami Mitwalli.

37.Al-Ahali 14/11/90, Hazim Munir.

38. Voir A. Roussillon op. cit.

39.Misr al-Fatat 26/11/90. 'Imad Subhi.

40. Rose al-Yusuf 26/11/90, Isam 'Abd al-'Aziz.

41.Al-Akhbar 28/11/90, 'Abd al-Salam Dawud

42.Al-Ahram 6/12/90, 'Abd al-Azim Darwich

43.Al-Ahram 19/11/90, 'Abd al-Azim Darwich

44.Al-Ahram 6/12/90, 'Abd al-Azim Darwich

45. Rose al-Yusuf 8/10/90, Muhammad Rizq p. 22.

46.Al-Ahram 2/11/90.

47.Al-Gumhuriyya 6/11/90, Mahmud Nafadi.

48.Al-Ahram 8/1/90.

49.Al-Gumhuriyya 8/11/90, Badawi Mahmud.

50.Al-Ahram 28/11/90, Ibrahim Nafi'.

51.Al-Ahrar 24/12/90, 'Adil Higazi.

52.op. cit.

53.Al-Ahram 19/12/90, Salah Muntasir

54.Al-Ahram 15/11/90, Iman Mustafa.

55.Akhbar EI-Yawm 8/12/90, Fatima Baraka.

56.op. cit.

57.Misr Al-Fatat 7/12/90, Kitah Ahmad

58.Al-Ahrar 24/12/90, 'Adil Higazi.

59.Al-Ahram 26/12/90, Ahmad Bahgat. 
60.Al-Akhbar 27/10/90, Husni Chah.

61.Sabah al-Khayr 8/11/90, Camélia Kamal al-Din, p. 8.

62.Sabah al-Khayr 8/11/90. Ali 'Abd al-Salam, p. 10-11.

63.Al-Ahram 1/11/90, Ahdaf al-Bandari.

64.Al-Ahram 13/11/90, Salama Ahmad Salama.

65.Al-Akhbar 9/12/90, 'Awatif al-Kilani.

66.Al-Wafd 8/11/90, Mahmud al-Charbini.

67.Al-Ahali 24/11/90, Mamduh Bichri Wisa.

68. Al-Ahali 21/11/90, Samir Tadros.

69.Al-Ahali 21/11/90, Salim Nagib.

70.Watani 25/11/90, Nabil 'Aziz 'Abd al-Malik.

71.Al-Ahali 19/12/90.

72.Al-Ahram 11/12/90.

73.Rose al-Yusuf 22/10/90, Hamdi 'Abd al-Aziz Gamal Taye' p. 27-31.

74.Al-Ahram 12/10/90.

75.Sabah al-Khayr 25/10/90, Hanan Gad, p. 14-15.

76.Al-Ahram 8/11/90, Izzat 'Abd al-Mun'im.

77.Al-Ahali 12/12/90, Hazim Munir.

78. Rose al-Yusuf 26/11/90, Sawsan al-Gayyar.

79.Al-Ahali 28/11/90, Mus'ad Nur, Mahmud al-Hadari.

80.Al-Ahram 30/10/90.

81.Al-Ahram 4/11/90.

82.Al-Akhbar 24/11/90, Muhyyi 'Abd al-Rahman.

83.Al-Wafd 21/11/90,Nasir Fayad.

84.Al-Akhbar 15/11/90.

85.Al-Ahram 19/11/90, Mahmud Mu'awad, p.18-23.

86.Al-Wafd 30/11/90.

87.Rose al-Yusuf 19/11/90. 'Abd Allah Kamal.

88.Al-Ahali 28/11/90, Mus'ad Nur Mahmud al-Hadari.

89.Al-Ahali 12/12/90, Sulayman Chafiq.

90.Al-Ahali 14/11/90. Midhat al-Zahid.

91.Al-Gumhuriyya 7/1/90.

92.Al-Ahali 12/12/90.

93.Al-Ahali 12/12/90, Phillipe Gallab.

94.Al-Wafd 31/12/90, Yusri Chabana.

95.Al-Wafd 7/12/90

96.Al-Musawwar 16/11/90, Faruq Abaza, p. 56-57.

97.Misr Al-Fatah 19/11/90.

98.Al-Ahali 21/11/90, Husayn Abd-Rabuh.

99.Al-Wafd 29/11/90.

100.Al-Ahali 28/11/90.

101.Al-Ahali $21 / 11 / 90$.

102.Al-Ahali $21 / 11 / 90$.

103.Al-Wafd 29/11/90, Mahmud al-Charbini.

104.Al-Wafd 13/1/91.

105.Al-Gumhuriyya 15/11/90, Badawi Mahmud.

106.Al-Siyasi 28/10/90, 'Abd al-Qadir al-Sa'dani.

107.Al-Wafd 6/12/90, Ayman Nur. 
108. Rose al-Yusuf 22/10/90, Hamdi 'Abd al-Aziz, Gamal Taye', p. 27-31.

109.Al-Ahali 28/11/90, Zayn al-Sammak.

110.Al-Ahram 12/11/90 Sayyid 'Ali.

111.Binder Léonard, in A moment of enthousiasm. The second stratum in Egypt, University of Chicago Press, Chicago, 1978.

112.Akhbar al-Yawm 8/12/90, Muhammad Imbabi.

113.Al-Wafd 6/12/90, Ayman Nur.

114.Al-Wafd $7 / 12^{\wedge} 0$.

115.Al-Wafd 16/12/90.

116.Rose al-Yusuf 19/11/90, Abd Allah Kamal, p. 29.

117.Al-Ahram 26/11/90, Dalal al-Atwi.

118.Al-Akhbar 10/10/90, Amru al-Khayyat.

119.Al-Wafd 10/10/90, Sami Sabri.

120.Al-Cha'b 2/11/90,MamduhQasim.

INDEX

Mots-clés : citoyenneté, clientélisme, démocratie, élections, fraude, PND, politique 